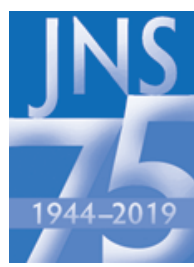

\title{
Surgical management of complex spinal cord lipomas: how, why, and when to operate. A review
}

\author{
JNSPG 75th Anniversary Invited Review Article
}

Dachling Pang, MD, FRCS(C), FRCS(Eng)

University of California, Davis, California; and Great Ormond Street Hospital for Children, NHS Trust, London, United Kingdom

\begin{abstract}
This review summarizes the classification, anatomy, and embryogenesis of complex spinal cord lipomas, and it describes in some detail the new technique of total lipoma resection and radical reconstruction of the affected neural placode. Its specific mission is to tackle two main issues surrounding the management of complex dysraphic lipomas: whether total resection confers better long-term benefits than partial resection and whether total resection fares better than conservative treatment-i.e., no surgery-for asymptomatic lipomas. Accordingly, the 24 -year progression-free survival data of the author and colleagues' series of over 300 cases of total resection are compared with historical data from multiple series (including the author and colleagues' own) of partial resection, and total resection data specifically for asymptomatic lesions are compared with the two known series of nonsurgical treatment of equivalent numbers of patients. These comparisons amply support the author's recommendation of total resection for most complex lipomas, with or without symptoms. The notable exception is the asymptomatic chaotic lipoma, whose peculiar anatomical relationship with the neural tissue defies even this aggressive surgical approach and consequently projects worse results (admittedly of a small number of cases) than for the other two lipoma subtypes of dorsal and transitional lesions. Prophylactic resection of asymptomatic chaotic lipomas is therefore not currently endorsed.
\end{abstract}

https://thejns.org/doi/abs/10.3171/2019.2.PEDS18390

KEYWORDS chaotic lipoma; complex spinal cord lipomas; intraoperative electrophysiological monitoring; long-term outcome; preoperative patient profiling; progression-free survival; spinal dysraphism; surgical technique; total resection; spine

$\mathrm{D}$ ESPITE recent advances in the treatment of dysraphic spinal cord lipomas, two issues concerning these lesions continue to take center stage in debates among pediatric neurosurgeons: one is whether asymptomatic lipomas should be treated with prophylactic surgery; the other is with what surgery the lesion should be treated.

Regarding the first issue, arguments continue unabated between the "cutters" (those who advocate early resection) and the "non-cutters" (those who would not do surgery until substantial signs of deficits appear), but one thing seems beyond dispute: spinal cord lipoma is a progressive disease. In 2004, Kulkarni et al. ${ }^{27}$ from L'hôpital Necker-Enfants malades, published a prospective study of a relatively large cohort of children with lipomas who were followed without surgery, and they concluded that asymptomatic lipomas have a 33\% chance of deterioration over 9 years. A comparable, though retrospective, study from London in $2012^{72}$ similarly reported a $40 \%$ 10-year deterioration rate in cases of unoperated asymptomatic lipomas. The London study also showed that females with transitional lipomas, especially those harboring a terminal syrinx, fared even worse. Thus, since half of their cohort was female and approximately $70 \%$ of their lipomas were of the transitional type, the poor outcome rate of the group, when projected onto a larger cohort size and longer followup period, may well exceed $40 \%$. Such dire statistics for untreated lipomas in children with presumably long actuarial survival seem to demand some type of intervention. Ironically, the Parisian group advised against prophylactic surgery because their own results of prophylactic partial resection in a later report showed an even higher probability of late deterioration than the group with no surgery. 27,62 The logical conclusion, considering the high progression

ABBREVIATIONS BCR = bulbocavernosus reflex; DREZ = dorsal root entry zone; EMG = electromyography; MCA = multiple correspondence analysis; ONTD = open neural tube defect; PFS = progression-free survival; TCMEP = transcortical motor evoked potential.

SUBMITTED January 26, 2019. ACCEPTED February 4, 2019.

INCLUDE WHEN CITING DOI: 10.3171/2019.2.PEDS18390. 
rates of both the natural disease and partial resection, is that asymptomatic lipomas do indeed need surgery, just not partial resection.

Which brings in the second issue regarding lipomas, of what is the safest and most effective surgery that offers long-term benefits over the natural history of the disease. Historically, the traditional technique for treating spinal cord lipomas had always been partial resection, $, 1,6,19,22,29,40,69$ but despite its widespread usage, data from most large series do not support the opinion that partial resection offers adequate long-term protection against disease progression. For example, Dorward et al. ${ }^{16}$ reported a symptomatic recurrence rate of $48 \%$ over merely 2.2 years. Colak et al. ${ }^{11}$ reported a 52\% recurrence rate over 10 years with partial resection, but their series comprised $37 \%$ terminal lipomas, which are known to have a much better prognosis than dorsal and transitional lipomas, implying that the progression rate, if calculated just for the latter lipoma types, must be even higher than 52\%. Pierre-Kahn and associates $^{61}$ documented a 10 -year recurrence of $46 \%$, but again their series contained many terminal and filar lipomas; Cochrane et al. $^{10}$ and Xenos et al. ${ }^{73}$ similarly recorded early recurrence. These disturbing results pose the obvious question of whether leaving behind a large amount of residual fat with its broad, raw, and sticky abraded surface actually provokes new adhesions to the adjacent dura mater and consequently incurs earlier and firmer retethering than if the lipoma had been left untouched, or, alternatively, whether complete removal of the bulky lipoma plus measures to render the now-pliant neural placode less "sticky" would impart greater permanent benefits than partial resection (note: the term neural placode in lipoma is borrowed from the main neural core of an open neural tube defect [ONTD] to emphasize its similar "neural" nature once the lipoma is removed. The synonymous usage of the term in lipoma and ONTD is logical if one compares the embryogenesis of the two entities [see below]; the "placode" in each case represents the original embryonic neural plate blighted in its final completing stage, having been invaded by paraxial mesenchyme in the lipoma and thwarted in its midline dorsal fusion in ONTD).

The need for a different procedure other than partial resection occurred to me in 1991, when my own series of 116 partial resections that followed conventional teaching $^{4,19,40,41,61,66,69}$ showed an alarming $65 \%$ symptomatic recurrence rate. This intuitively untenable situation, abetted by hordes of agonizing and complaining patients, compelled me to entertain the then iconoclastic concept of complete lipoma resection, strategic reconstruction of the neural placode, and expansile duraplasty. My rationale for this more aggressive approach ${ }^{58}$ was founded on three hypotheses: 1) the high recurrence rate after partial resection is due to retethering at the resection site; 2) retethering is in turn promoted by three conditions: a tight content-container relationship between the affected placode and its adjacent intradural space, a broad "sticky" nidus of remaining fat, and incomplete separation of the terminal placode from the caudal fat (see below); and 3) total resection eliminates these conditions contributing to retethering and thus reduces the probability of late deterioration. The objective of this proposed new operation is therefore to create a milieu least conducive to resticking of the neural placode. The first premise toward this goal is predicated on the observation that the spinal cord normally shows considerable intradural motion to gravity and postural changes shown on dynamic imaging. ${ }^{5,15}$ Lowering the content-container ratio and amplifying the amount of free placode movements within the dural sac should therefore reduce sustained contacts between placode and dura and, hence, also the probability of adhesion. To achieve this in a "virgin" (previously untouched) lipoma, the bulk of the lipoma-cord composite must first be severely trimmed by resecting all or most of the engorging and unwieldy fat down to the thin, supple neural placode; for "redo" lesions that have undergone previous surgery, the stiff fibrous scar must also be resected. The goal is to render the thinnest, most malleable neural placode so that through tensionless pia-to-pia neurulation (see below), a slender but unstrangled pia-covered neural tube can be created. The sticky lipoma bed is thus concealed within this tube, and the dural sac is then made capacious by a generous graft. Finally, total elimination of the obscuring fat will also enhance the chances of terminal untethering.

In 2009 and 2010,58,59 my colleagues and I published our early series on 238 patients who underwent total/neartotal resection of complex lipomas, and we compared their short- and long-term outcomes with our prior series of 116 patients in whom partial resection was performed. In 2013, we updated our series of total resections to 315 cases ${ }^{60}$ and, recently, our number has risen to over 420 cases. ${ }^{54}$ Our ever-enlarging data pool unfailingly shows an overwhelming advantage of total over partial resection in almost all respects. A number of pediatric neurosurgeons have since visited us to learn the technique and have converted to total resection for symptomatic lipomas, but the subset of asymptomatic virgin lipomas continues to pique uncertainties and controversies. This review therefore reexamines 3 recurring questions: 1) Is total resection better than partial resection for both symptomatic and asymptomatic lipomas? 2) Is total resection better than no surgery for asymptomatic lipomas, i.e., should they all be treated prophylactically with total resection? 3) Is partial resection worse than no surgery and, if so, should it be strongly discouraged?

For utility purposes, I also intend this review to serve as a sort of surgical vade mecum and include sections on practical anatomy and embryology, helpful information concerning intraoperative neurophysiological monitoring, and a rather detailed illustrated exposition of the technique of total lipoma resection.

\section{Anatomy and Classification}

In the literature, the nomenclature and classification of spinal cord lipomas are imprecise and inconsistent. Here, we define the types of lipomas as follows:

\section{Dorsal Lipoma}

A dorsal lipoma perches entirely on the dorsal surface of the lumbar spinal cord and never involves the conus (Fig. 1). The junction between the lipoma, spinal cord, and pia, the fusion line (see below), is usually traceable along 


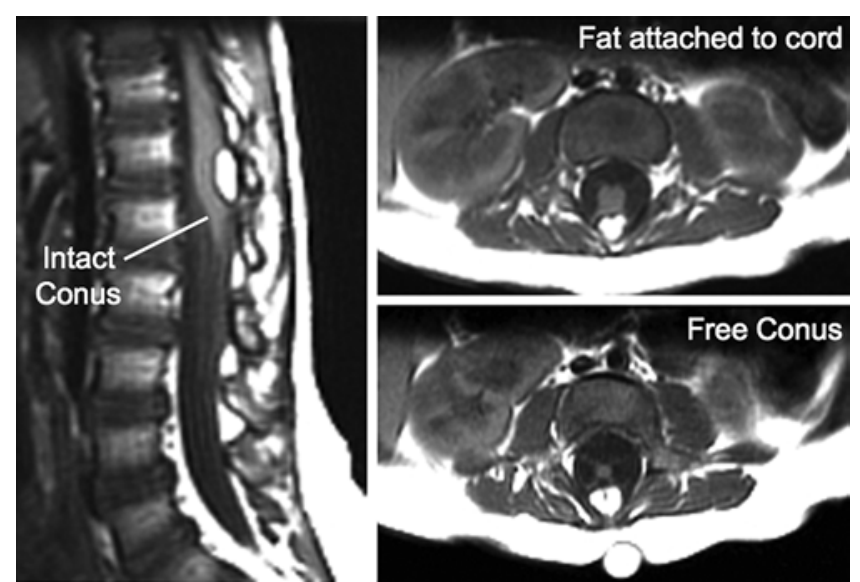

FIG. 1. Dorsal lipoma on MRI. Left: Sagittal image shows intact conus caudal to lipoma stalk. Axial images demonstrating site of lipoma attachment to cord (upper right) and free conus just caudal to the level of lipoma attachment (lower right). Reprinted from: Pang D et al: Surgical treatment of complex spinal cord lipomas. Childs Nerv Syst (29):14851513, 2013. Published with Permission from Springer Nature.

a roughly oval track, demarcating fat from the more lateral dorsal root entry zone (DREZ) and nerve roots (Fig. 2 and Supplemental Fig. 1A), which means the nerve roots and DREZ are never mixed up with the lipoma. The lipomatous stalk usually traverses a fairly discrete defect in the dorsal dura to blend with the subcutaneous fat. The uninvolved conus medullaris often ends in a thickened filum terminale. An idealized depiction of the resection of a typical dorsal lipoma is shown in Supplemental Fig. 1B, which offers a clear view of the entire circumference of the fusion line and how the surgeon can command a full $360^{\circ}$ approach to the lipoma-cord interface.

\section{Transitional Lipoma}

The rostral part of a transitional lipoma resembles a dorsal lipoma in that it features a discrete fusion line and a reasonably organized array of nerve roots and DREZ, but unlike the dorsal type, the fat plane of a transitional lipoma then cuts ventrocaudally to involve the entire conus, likened to making an oblique, bevelled knife-cut across the end of a rod (Fig. 3). Also, unlike the dorsal type, its lipoma-cord interface is often not horizontally leveled, and the neural placode may be so rotated in its longitudinal axis that it literally spins into a parasagittal orientation, but the neural tissue is always recognizable ventral to this interface, so that the DREZ and nerve roots are lateral and ventral to the fusion line and therefore also do not usually traverse the lipoma (Supplemental Fig. 2A). An idealized depiction of a total resection of a transitional lipoma thus shows a ventrally slanting oblique plane of resection set by the fusion line (Supplemental Fig. 2B). A filum may or may not be present, and the dural defect often extends way past the neural placode to involve the caudal dural sac.

In general, dorsal lipomas conform to the standard architecture described above and are mostly horizontal and symmetrical. Transitional lipomas, on the other hand, tend more to be irregular and unpredictable: many are not symmetrical or flat, and the larger ones often overhang the nerve roots and require special handling; congenitally de-

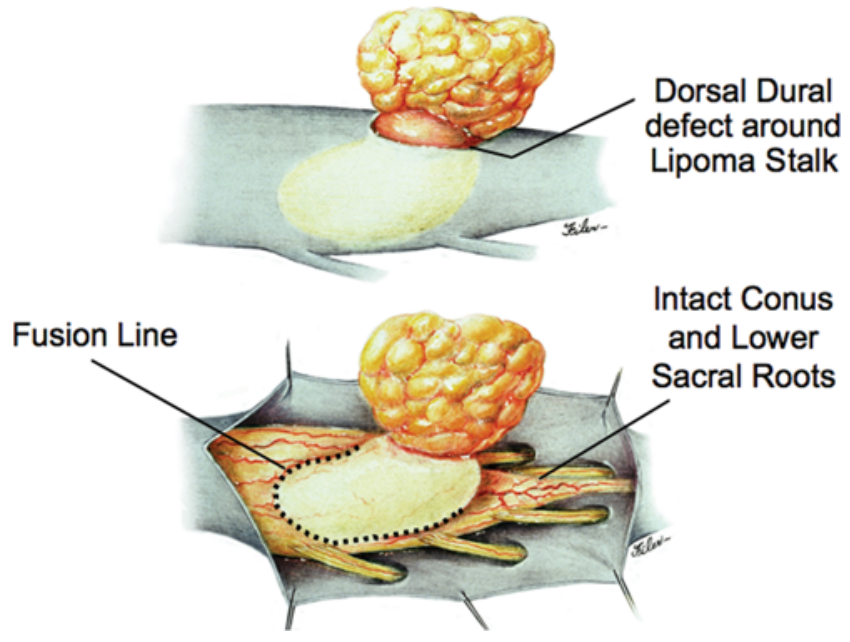

FIG. 2. Intraoperative drawings of a dorsal lipoma: neat dorsal dural defect through which lipoma stalk goes (upper) and a circumferential fusion line and intact conus (lower). Reprinted from: Pang D et al: Surgical treatment of complex spinal cord lipomas. Childs Nerv Syst (29):14851513,2013 . Published with Permission from Springer Nature.

funct nerve roots do sometimes run through the lipoma and must be correctly recognized and dispensed with; and monstrous transitional lipomas have been known to overwhelm any earnest attempt to sort out the "good" anatomy.

\section{Terminal Lipoma}

All terminal lipomas are sharply attached across the base of the conus without ever involving the dorsal parts of the spinal cord or nerve roots. Thus, the lipoma-cord interface lies entirely below all the functional sacral roots and conus, which essentially looks normal except for the attached fat at the end. The dural sac and the overlying myofascial layers are intact. The lipoma either replaces

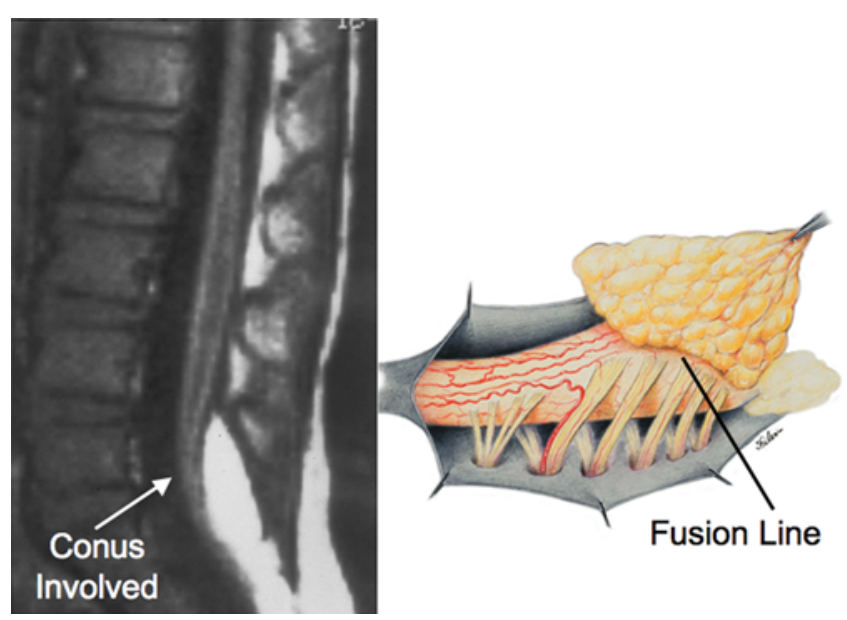

FIG. 3. Transitional lipoma. Left: Sagittal MR image showing that a lipoma begins dorsally but involves the entire conus. Ventral side of neural placode is free of fat. Right: The plane of the fusion line begins dorsally and then cuts obliquely toward the tip of the conus. The array of the DREZ and dorsal roots is also forced to slant dorsoventrally. Reprinted from: Pang D et al: Surgical treatment of complex spinal cord lipomas. Childs Nerv Syst (29):1485-1513, 2013. Published with Permission from Springer Nature. 
the filum entirely, or is embedded within a portion of the filum, in which case it is often called a filar lipoma.

The excision of terminal lipoma is relatively simple and its prognosis is far better than that of the other types; both are beyond the scope of this review.

\section{Chaotic Lipoma}

This novel type that the author and colleagues introduced in $2009^{58}$ is so named because neither its embryogenesis nor its anatomy follows the rules of the other lipoma types. Its rostral portion may look disarmingly orthodox, like the dorsal or transitional lipoma, but the fat of its caudal portion percolates through the neural placode to its ventral side and always engulfs neural tissue and nerve roots to some degree (Supplemental Fig. 3A). The fusion line may be visible in its rostrodorsal portion but becomes undefinable caudally as it vanishes behind the ventral fat, where the DREZ and nerve roots are no longer easily identifiable. The moniker "chaotic" depicts the confusing blend of ventral fat and neural placode, and the often-impossible task of differentiating the wandering fat from the invisible neural tissue and nerve roots at surgery (Supplemental Fig. 3B). Chaotic lipomas are uncommon but are typically associated with sacral agenesis. ${ }^{58}$

The literature ${ }^{1,62}$ mentions one other lipoma type, the lipomyelomeningocele, in which the caudal spinal cord and parts of the lipoma extrude dorsally out of the spinal canal, together with a an outpouching of the cerebrospinal fluid (CSF) sac (Supplemental Fig. 4). The basic configuration of the lipoma is not different from that of a conventional transitional or dorsal lesion. Accordingly, we choose to include this variant subtype as either a transitional or dorsal lipoma with a descriptive qualifier of "extraspinal extension."

\section{Surgically Relevant Embryology}

An understanding of the embryogenesis of lipomas is helpful in appreciating the surgical nuances.

\section{Embryogenesis of Dorsal and Transitional Lipomas}

In the human embryo, a progressive longitudinal disparity develops between the spinal cord and vertebral column because of their unequal growth rates. ${ }^{14,17,28,68}$ Allowing for some shortening of the caudal neural tube by programmed apoptosis during secondary neurulation, the tip of the cord is still noted to have ascended a fair distance from opposite the coccyx in the 30-mm embryo to approximately the L2 level at birth. ${ }^{3,23,28,68}$ Smooth upward movement requires a well-constructed neural tube within a smooth dural tunnel. If during the ascent a dorsal defect develops in the dura and neural tube, the surrounding mesenchyme will invade through the defect and form a fibrofatty stalk that attaches to the sliding neural tube to cause its entrapment. This embryogenic sequence implies a focal failure in neural tube closure during primary neurulation (secondary neurulation does not involve neural folds fusion) and thus applies only to the dorsal and transitional lipomas (see below). It also explains why these two lipoma types are always associated with adjacent bifid neural arches.

Before completion of primary neurulation, the cutane- ous and neural ectoderms must first separate from each other, but this process, called disjunction, does not occur until after the midline fusion of the dorsal neural folds. This time sequence is important because it guarantees exclusion of the extraneural mesenchyme from ever entering the neural tube to make contact with its inner (ependymal) surface. The embryological error leading to the mesenchymal invasion of the sliding neural tube probably lies in premature disjunction between the two ectoderms $\mathrm{s}^{7,43}$ i.e., ectodermal separation occurs before fusion of the converging neural folds, leaving a small gap next to the open neural tube through which paraxial mesenchyme can migrate into the central canal. Once such a mesenchymal stalk forms through this gap, further closure of the neural tube is prevented and a segmental dorsal myeloschisis is created (Fig. 4A and B). Alternatively, the inversion of the fusion-disjunction sequence can also be caused by incompetence of the paraxial mesoderm in forcing timely convergence of the opposing neural folds, delaying their fusion and allowing disjunction to happen first. ${ }^{30-36}$ Lastly, metabolic disturbance of the cell membrane-bound glycosaminoglycans at the edges of the neural folds, which are vital to cell-cell recognition and adhesion, $39,44,47,71$ could likewise delay neural folds fusion and reverse the sequence.

Pluripotential mesenchyme has been shown to form diverse derivatives depending on the kind of inducers secreted by the adjacent neuroectoderm ${ }^{13,24}$ (Fig. 4C). Once exposed to the invading mesenchyme, the ependymal lining of the neural tube has the inherent propensity to induce it to form fat, muscles, collagen, and even bone and cartilage (Supplemental Fig. 5). In contrast, the outer surface of the neural tube normally initiates the production of meninges. ${ }^{42}$ However, new meninges cannot now form across the dorsal midline because of the impeding lipoma stalk in-evolution, which ultimately tethers the neural tube to the subcutaneous fat. Similarly, defects in the developing myofascial structures (from myotomal mesoderm) and neural arches (from scleromesoderm) also neatly surround the lipoma stalk (Fig. 4D).

Once formed, the mesenchyme-derived fat and collagen within the neural tube fuse with the developing alar and basal plates. Because the dorsal root ganglions descend from neural crest cells pinched from the external surface of the neural folds just lateral to their site of failed fusion, their central processes, the dorsal nerve roots, also enter the spinal cord lateral to, but never traversing, the lipoma stalk. Their entry zone (the DREZ) must correspondingly lie very near, but always lateral, to the exact junctional boundary between the lipoma and the spinal cord. Recognizing and understanding this boundary, called the fusion line, is essential before undertaking the actual resection of the lipoma ${ }^{51,52,58}$ (Fig. 4D). Meanwhile, the "disjoined" cutaneous ectoderm crosses over the chaos underneath to form wholesome skin above, although minor perturbation in the process may produce subtle signatures, such as a dimple, crater, or capillary hemangioma overlying the subcutaneous lipoma.

The embryogenesis of dorsal lipoma perfectly exemplifies mistimed disjunction during primary neurulation. Its fatty stalk only involves cord segments above the conus, 


\section{Embryogenesis of Dorsal Lipoma}

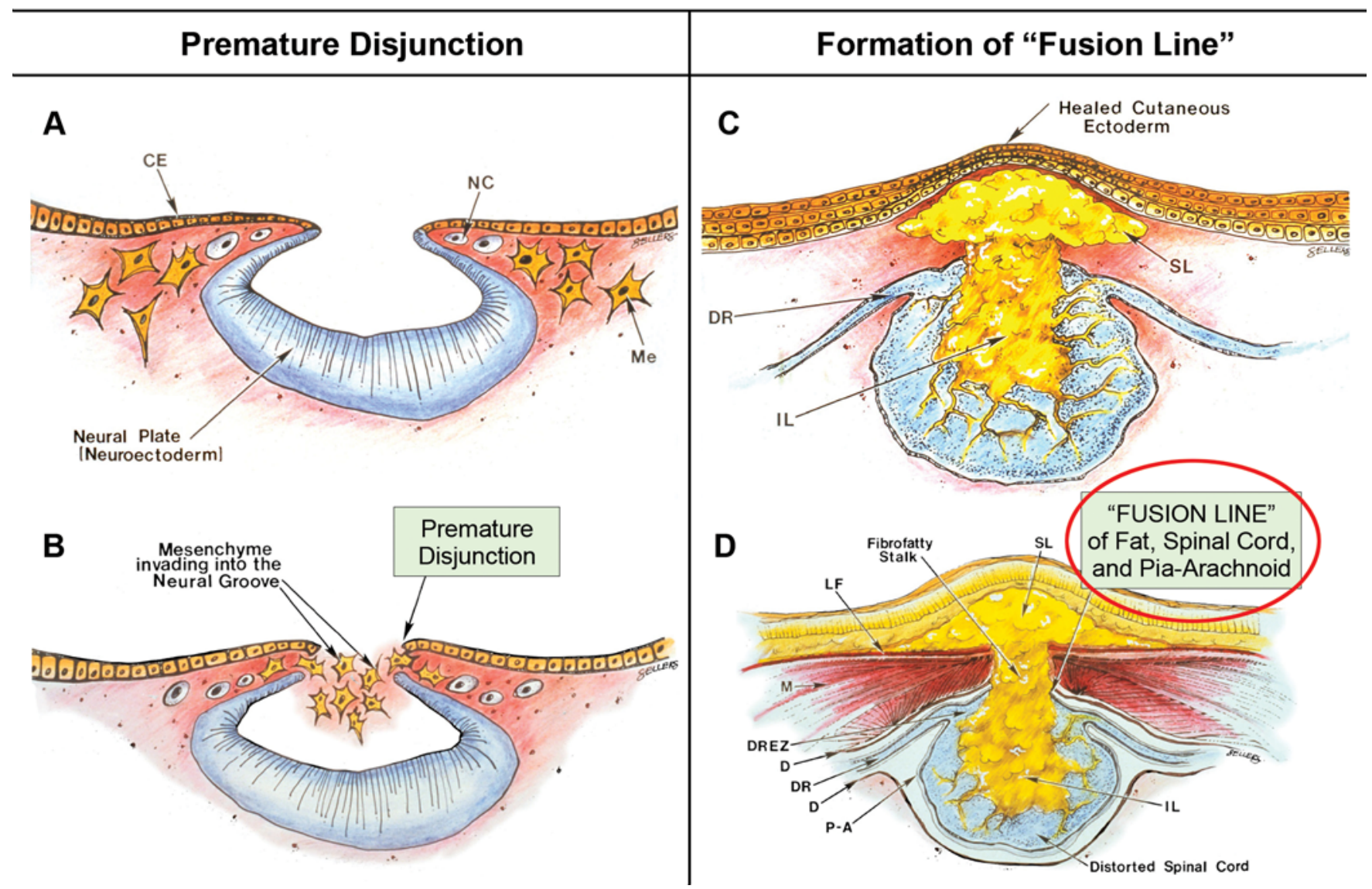

FIG. 4. Embryogenesis of dorsal lipoma, a pure primary neurulation defect. A and B: Premature disjunction before complete closure of neural plates allows migration of mesenchymal cells into neural groove to establish contact with the ependymal surface. C and D: Formation of fusion line between lipoma, cord, and pia-arachnoid. DREZ and dorsal root are always lateral to the fusion line and thus not entangled in fat. $C E=$ cutaneous ectoderm; $D=$ dura; $D R=$ dorsal root; $I L=$ intramedullary lipoma; $L F$ = lumbodorsal fascia; $\mathrm{M}=$ muscle; $\mathrm{Me}=$ mesenchyme; $\mathrm{NC}=$ neural crest; $\mathrm{P}-\mathrm{A}=$ pia-arachnoid; $\mathrm{SL}=$ subcutaneous lipoma. Reprinted from Pang D: Total resection of complex spinal cord lipomas: how, why, and when to operate. Neurol Med Chir (Tokyo) 55: 695-721, 2015. Published with permission from the Japanese Neurosurgical Society. CC-BY-NC-ND (https://creativecommons. org/licenses/by-nc-nd/4.0/deed.ja).

the latter being a product of secondary neurulation. Furthermore, premature disjunction of the converging neural folds is always segmental, meaning proper disjunction and closure take place business-as-usual both immediately rostral and caudal to the faulty focus. This "square pulse" timeline of the abnormal event in dorsal lipoma therefore results in a sharply demarcated fusion line both rostral and caudal to the lipomatous stalk ${ }^{51-53,58}$ (Fig. 2, Supplemental Fig. 1A and B). Dorsal lipomas thus exist only on parts of the matured spinal cord formed from the primary neural tube, and they represent only $15 \%$ of lipomas in our series. ${ }^{58-60}$

In transitional lipoma, faulty development involves much more than simple mistimed disjunction in an isolated segment of the primary neural tube. Even though its rostral part resembles the dorsal lipoma, the fatty invasion of the whole of the conus means that not only primary but also secondary neurulation has been profoundly disturbed. This is evidenced by the frequent incorporation of the filum, an undisputed remnant of late secondary neu- rulation, into the transitional lipoma, and that vacuoles resembling the cavitary spaces typically seen in the medullary cord during mid-secondary neurulation, are found in abundance within the core of the lipoma (see below). Also, while the rostral part of the transitional lipoma only affects the dorsal surface of the lumbar cord, the distal part involves the entire core of the conus, strongly suggestive of aberrant inclusion of wayward mesenchyme into the developing conus during the condensation phase of secondary neurulation. Intramedullary mesenchyme may then migrate up and down the neural tube within the conjoint primary and secondary neural canals. ${ }^{65}$ The hypothesis that the rostral half of the transitional lipoma comes from faulty primary neural tube fusion affecting only the superficial layers of the lumbar cord, and its caudal half from abnormal condensation within the deeper core of the secondary neural tube, may well explain the oblique ventral-caudal slant of its lipoma-cord interface. The far less confined and "non-square pulse" nature of its embryogenetic error also explains why the fusion line of 
a transitional lesion is never as well defined and leveled as that of the dorsal lipoma, often has no caudal demarcation, and its fatty acreage is a great deal more sprawling. In spite of the latter, the lipoma-cord interface remains relatively distinct in most transitional lipomas.

\section{Embryogenesis of Chaotic Lipomas}

Chaotic lipomas do not not quite fit into the scheme for either the dorsal or transitional type. They may have a rostral part half-resembling a dorsal lipoma, but caudally there is no definable lipoma-cord interface, and the fat percolates through the whole thickness of the neural placode to its ventral surface in large and unruly measures. The confusing interplay between fat and cord in this type of lipoma seems to be in constant chaos.

Embryologically, the obvious dissimilarities between the features of a chaotic lipoma and the familiar consequences of mistimed disjunction, as well as its strong association with sacral agenesis $\left(82 \%\right.$ in our 2009 series $\left.^{58}\right)$, suggest that its origin may be part of a general failure of the caudal cell mass during early secondary neurulation (Supplemental Fig. 6). ${ }^{45,46}$ Secondary neurulation comprises 3 distinct stages: 1) condensation and transformation of pluripotential stem cells from the caudal cell mass into committed neural progenitor cells to form the solid medullary cord; 2) intrachordal cavitation of the medullary cord, ${ }^{46,64,65}$ followed by its integration with the primary neural tube; and 3) partial dissolution of the cavitary medullary cord by programmed apoptosis to form the filum terminale. ${ }^{28,65}$ In this context, chaotic lipoma probably results from an aberrant preponderance and accelerated differentiation of lipogenic stem cells from the caudal cell mass during early medullary cord condensation, leading to inclusion of lipoid cells within the neural progenitor cell pool, destined to beget a distorted conus permeated with fat through and through. ${ }^{58}$

\section{Embryogenesis of Terminal Lipoma}

Terminal lipomas involve only the conus and never the lumbar or upper sacral cord, strong evidence that they arise from faulty secondary rather than primary neurulation. Furthermore, defects in the lumbodorsal fascia, dura, and dorsal spinal cord, all hallmarks of failed primary neural tube closure, are never seen with terminal lipomas. Lastly, terminal lipomas either replace or are embedded in portions of the filum, which temporally and topographically places their pathogenesis within the period of secondary neural tube formation. The fact that their distal conus always appears well formed argues against disrupted condensation of the medullary cord in early secondary neurulation. Rather, the almost obligatory presence of disorganized neuroglia and ependymal tubules within terminal lipomas ${ }^{70}$ suggests, instead, an incompetent apoptotic machinery during the late degenerative stage of secondary neurulation as the basic pathogenetic mechanism. ${ }^{57}$

\section{Intraoperative Electrophysiological Monitoring}

Intraoperative electrophysiological monitoring is sine qua non in lipoma surgery. ${ }^{49,50,55,58,60}$

\section{Electromyographic Needle Placements}

A comprehensive electromyography (EMG) system is set up to capture triggered responses from muscles supplied by the lumbosacral nerve roots. Standard 27-gauge EMG needle electrodes are inserted into the rectus femorus (L4), anterior tibialis (L4-5), gastrocnemius (S1), and abductor hallucis (S2). A pair of smaller-gauge (no. 29) EMG needles are inserted obliquely into the external anal sphincter at the anal verge on each side. A plug of dry muslin gauze is pushed halfway into the anal canal to isolate the sphincter contractions of one side from the other. ${ }^{49} \mathrm{In}$ addition, EMG measurements are made from the abductor pollicis brevis in each hand as a monitor for equipment malfunction. All stimulations and EMG recordings in the author's unit are done with the Cascade Intraoperative Monitoring System (Cadwell Laboratories, Inc.) using the Cascade Software version 2.5, but several other system brands on the market are equally proficient.

\section{Bulbocavernosus Reflex}

This is the "electrical" version of the reflexive contraction of the external anal sphincter when the glans penis is firmly squeezed and quickly released. For the afferent sensory arm of the reflex, pairs of pad or needle electrodes are placed on the sides of the penile shaft in males and between the labia minora and the periclitoral skin in females. Stimulation of the somatic sensory domain of the pudendal nerve via these electrodes generates a reflexive contraction of the external anal sphincter measurable by EMG. The bulbocavernosus reflex (BCR) is therefore a form of $\mathrm{H}$ reflex within the conus useful in monitoring the integrity of the central sensorimotor connections of the sacral cord segments. ${ }^{49,50}$

Special comments need to be made about the BCR, which is still very much a work-in-progress. The pudendal somatic sensory-pudendal somatic motor arc-i.e., the basic BCR circuit-is bilateral, meaning that stimulation on one side will generate sphincter contractions on both sides. The response is highly stimulation-parameter dependent. We use a train of 4 stimuli to capitalize on the powerful principle of post-tetanic potentiation and fairly reliably obtain motor responses from the anus. However, the BCR is highly sensitive to inhalation anesthetic concentrations, so that a sevoflurane level of higher than 0.5 MAC may disrupt it. The BCR is also uncertain in infants younger than 6 months so that allowances must be made for its inconstancy in newborn cases. But most of all, it must be clearly stipulated that the actual neuroelectrical circuit responsible for the BCR involves somatic sensory inputs from the perineal skin corresponding to the dermatomes of $S_{2}$ to $S_{4}$ via the pudendal sensory fibers, and somatic motor neurons within the anterior horn (Onuf's nucleus) projecting out via the pudendal motor fibers to the external anal sphincter. On the other hand, the sacral bladder circuit involves afferent visceral sensory fibers from the bladder wall stretch receptors via the pelvic nerve, and the efferent visceral motor neurons to the bladder detrusor muscles that reside within the autonomic (visceral) intermediolateral column of the central gray core of the sacral spinal cord, projecting out via the parasympathetic pelvic nerve. Thus, the anatomical pathways for the BCR and 
for the bladder voiding reflex are located in different, albeit neighboring, parts of the spinal cord, though both are within the S2-4 cord segments. Theoretically, a disrupted BCR during surgery does not necessarily imply damage to the bladder wall tension-detrusor reflex function, although frequently the prediction is true. A correlative study of the two is needed to sort out the BCR's true value.

\section{Nerve Root Stimulation and Direct Spinal Cord Stimulation}

Triggered EMG activity is obtained from direct stimulation of the motor roots and spinal cord using the concentric coaxial bipolar stimulation probe with a small tip diameter of $1.75 \mathrm{~mm}$ (Medtronic Xomed, Inc.) (Supplemental Fig. 7). The concentric configuration and small sizes of the anode-cathode rings permit delivery of extremely focused current to a very small target volume, ideal for selective activation of small and crowded electroresponsive units, such as the delicate sacral rootlets and the compact anterior horn of the conus. ${ }^{58}$ Stimulating currents from 0.3 to 5.0 $\mathrm{mA}$ are used, depending on target impedance. Most functional motor roots can be triggered by currents of $0.3-1.0$ $\mathrm{mA}$, although sometimes $1.5 \mathrm{~mA}$ is needed if the roots are partially fibrotic or wrapped up in arachnoid adhesions. Direct spinal cord stimulation will require a current of $2.0 \mathrm{~mA}$ or higher but seldom more than $5 \mathrm{~mA}$. Both sides will be recruited if the probe is placed on the midline, but a biased ipsilateral response can be elicited if the probe is shifted to a point ventral to the equatorial plane of the cord's coronal aspect. The stimulation frequency is usually 10 per second. Noting the rhythmic nature of the triggered contractions will distinguish them from the random spontaneous firing caused by surgical manipulation.

\section{Transcortical Motor Evoked Potentials}

In recent years, we have been able to reliably obtain continuous monitoring of transcortical motor evoked potentials (TcMEPs) from the external anal sphincter. The stimulation parameters are the same as for the lower limbs: a train of 8 pulses, each with a duration of $75 \mu \mathrm{sec}$ and an intensity of 100-300 V. In adults and children older than 2 years of age, 150-225 V are generally adequate but in infants, the stimulation intensity may have to be raised to 350 $\mathrm{V}$, presumably because of the poorly myelinated motor cortex and its higher impedance, only partially compensated for by the thin cranium. The inhalation anesthetic concentration, e.g., of sevoflurane, is usually set no higher than 0.5 MAC to ensure a high response rate, or, failing that, abandoned completely for total intravenous anesthesia in some infants and young children. Given that the BCR can be variable or even unobtainable in infants, TcMEP is an important modality for assessing the integrity of the central motor circuits of the sacral spinal cord.

\section{Somatosensory Evoked Potentials}

Stimulating electrodes either of the pad or needle type are placed over the course of the posterior tibial nerve behind the medial malleolus and over the common peroneal nerve at the fibular neck to provide the somatic sensory inputs for somatosensory evoked potential monitoring of the spinal cord segments above S2. ${ }^{49}$ It should be empha- sized that the sensory inputs from both these nerves are going into the L5-S1 cord segments, considerably higher than the usual location of most lipomas, so disturbance of conduction in the S2-4 sensory roots will therefore not affect the somatosensory evoked potentials.

\section{Surgical Technique of Total/Near-Total Lipoma Resection}

The surgical technique described below concerns the case of an unusually large and rambling dorsal lipoma that illustrates most of the key technical points of total resection applicable to both dorsal and transitional lipomas. The case involved a 12-month-old girl who was neurologically normal at birth but underwent MRI because of a lumbosacral fatty lump. MRI showed a moderate-size dorsal lipoma involving the lower lumbar cord. At 1 year, she began showing left leg weakness, and repeat MRI revealed massive enlargement of the lipoma (Supplemental Fig. 8).

\section{Step 1. Exposure and Dealing With the Extraspinal Lipoma Stalk}

The upper extent of the skin incision should be about 2 $\mathrm{cm}$ above the rostral end of the lipoma. The lower extent ideally includes at least $1 \mathrm{~cm}$ beyond the tip of the conus in a dorsal lipoma or of the neural placode in a transitional lipoma. Two metal skin markers are used to mark the longitudinal span of the planned laminectomies for a lateral radiograph, which is then matched up with the sagittal MR image to finalize the skin incision (Supplemental Fig. 9).

During the soft-tissue exposure, temptation to remove the subcutaneous lipoma must be resisted to avoid creating a tense fluid-filled dead space afterwards, which may compromise wound healing. Frequently, a relatively robust fatty stalk slightly more compact than the loose subcutaneous adiposity can be discretely traced through a defect in the lumbodorsal fascia with minimal dissection (Supplemental Fig. 10). This fatty stalk is a signpost to the lipoma's attachment on the spinal cord below and must therefore be handled with care during fascial exposure.

The amount of proximal bone removal must be adequate to allow for an unencumbered view of the upper tip of the lipoma, where the last set of normal nerve roots and the DREZ are used as anatomical landmarks, but more importantly, wide bilateral laminectomy down to the pedicles must be done to gain access to the far-lateral corners of the dural tube (see below). Exposure of some normal dura proximal to the lipoma also provides a visual perspective of how much the neural placode had protruded beyond the spinal canal in lipomas with extraspinal extension. The heavy mound of fat attached to the fatty stalk can now be removed to avoid inadvertent tugging on the spinal cord (Supplemental Fig. 10).

\section{Step 2. Detachment of Lipoma From Dura: Unhinging the Hammock}

The dura is opened in the midline about $1 \mathrm{~cm}$ rostral to the upper end of the lipoma, identified by its slight yellow tinge below the pia. Tight and closely spaced dural tagging sutures are placed to gain maximum exposure of the far-lateral alcoves of the dural tube afforded by the 


\section{Lipoma Suspended at Dural Adhesion Points Like a Hammock}
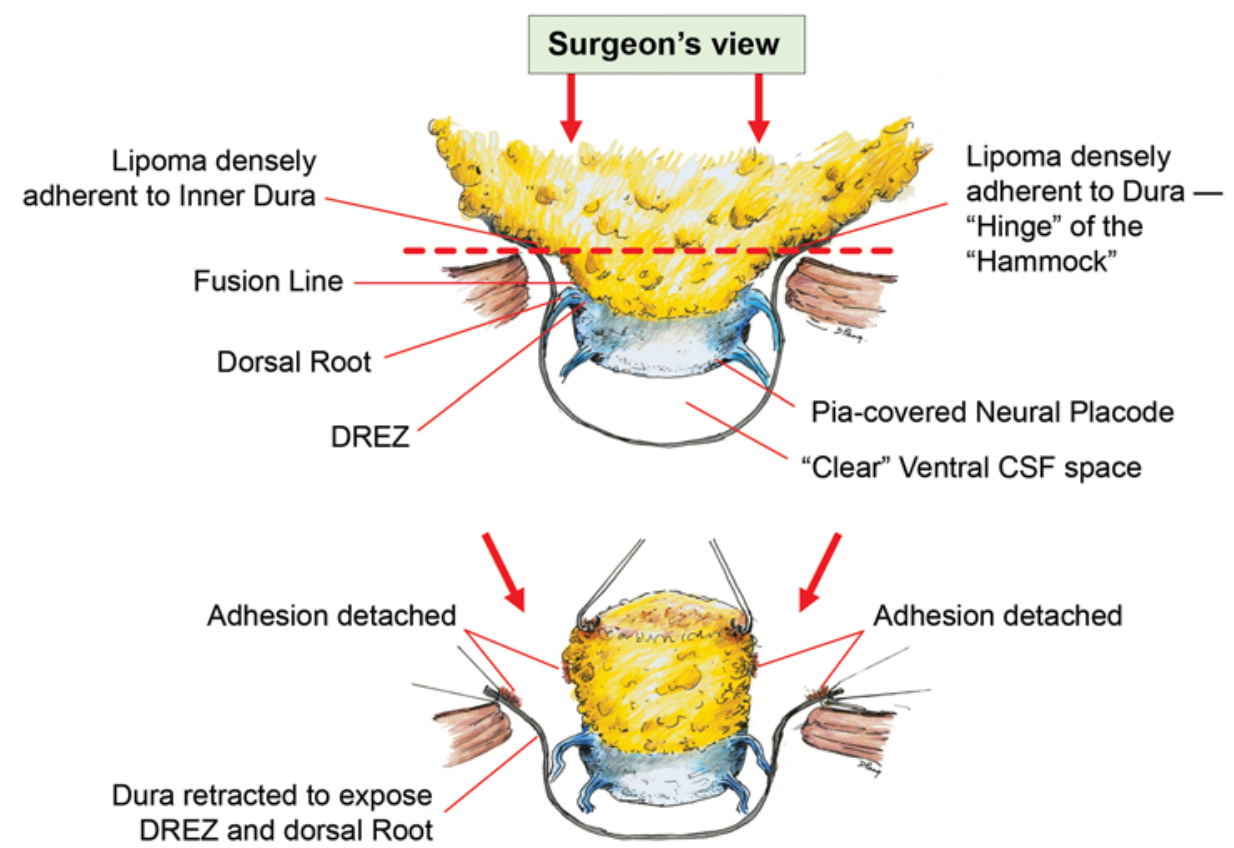

FIG. 5. Drawing depicting the relationships among the lipoma, neural placode, nerve roots, and dural sac in an axial slice. Upper: The lipoma-cord assembly is suspended at the dural edges at far-lateral adhesion points like a hammock against side hinges. The dotted transverse line that joins the two side hinges divides the assembly into a dorsal disorderly, fibrofatty half that completely blocks the surgeon's view to a much more orderly ventral half, containing the important anatomical landmarks of fusion line, DREZ, dorsal roots, fat-free ventral placode, and pristine ventral CSF space. Lower: After detaching the far-lateral adhesion points (the hinges) by careful "crotch dissection," and folding in the fatty mass, the ventral anatomical landmarks can now be visualized. Reprinted from Pang D: Total resection of complex spinal cord lipomas: how, why, and when to operate. Neurol Med Chir (Tokyo) 55: 695-721, 2015. Published with permission from the Japanese Neurosurgical Society. CC-BY-NC-ND (https://creativecommons. org/licenses/by-nc-nd/4.0/deed.ja).

wide laminectomy. This is a crucial but often neglected maneuver, because tagging the dural edge helps to reveal the "crotch" at the embryological fusion line, where the lateral fringe of the lipoma is attached to the inner edges of the dural defect. The critical importance of adequately exposing the crotch is shown best by examining any given axial slice of the lesion, in which the lipoma is roughly divided by a transverse line joining the site of lipoma-dura attachment, i.e., the so-called crotch, on each side. The entire lipoma-cord assembly is, in effect, suspended from the dural ceiling like a hammock at these two crotches. From a dorsal perspective, the surgeon thus has no visualization whatsoever of any meaningful anatomy ventral to this transverse line, such as the dorsal roots, DREZ, neural placode, or CSF pool (see Fig. 5 upper) until this hammock can be unsuspended by releasing the two crotch hinges. The lateral edges of the neural placode and nerve roots can then be folded inward enough to be identified and preserved (Fig. 5 lower).

Safe unhinging of the hammock must begin at the rostral extent of the crotch line where there is at least a semblance of normal anatomy. The "crotch" is kept under tension by pulling the fat firmly away from the dura, and its attachment to the inner dural lining minutely cut literally a millimeter at a time to avoid injuring the dorsal roots on the DREZ, which are very close to the crotch only millimeters below the fat (Supplemental Fig. 11). These initially hidden roots should pop into view as more of the fatty hammock is peeled back (Supplemental Fig. 12A and B), and any binding adhesions to the arachnoid can be gently dissected away (Supplemental Fig. 13). The clean ventral surface of the neural placode is now seen to advantage (Supplemental Fig. 14). This laborious but indispensable and ultimately rewarding step of crotch dissection is carried caudally until all the functioning nerve roots are revealed and the entire lipoma-neural placode assembly is completely unsuspended and freed from the dura (Fig. 6).

Preparing the crotch for dissection is sometimes difficult. If the dura is so attenuated that it clings to the side of the lipoma and spinal cord like pia mater, as on the left side here, the surgeon can lose perspective and mistakenly stray into the extradural space, where the nerve roots are entangled in stringy extradural fat and can be accidentally injured. When caught in this predicament, it is best to abandon further dissection on the "bad" left side and pick up a brisker pace by opening the crotch on the less problematic right side. After the crotch adhesions here are dispatched with and the free ventral subarachnoid space is entered, the collapsed left subarachnoid space can now be confidently accessed by navigating under the neural placode, and the left crotch dissection can finally be accomplished (Supplemental Fig. 15).

\section{Step 3. Lipoma Resection}

Before the actual lipoma resection, the fusion line must 


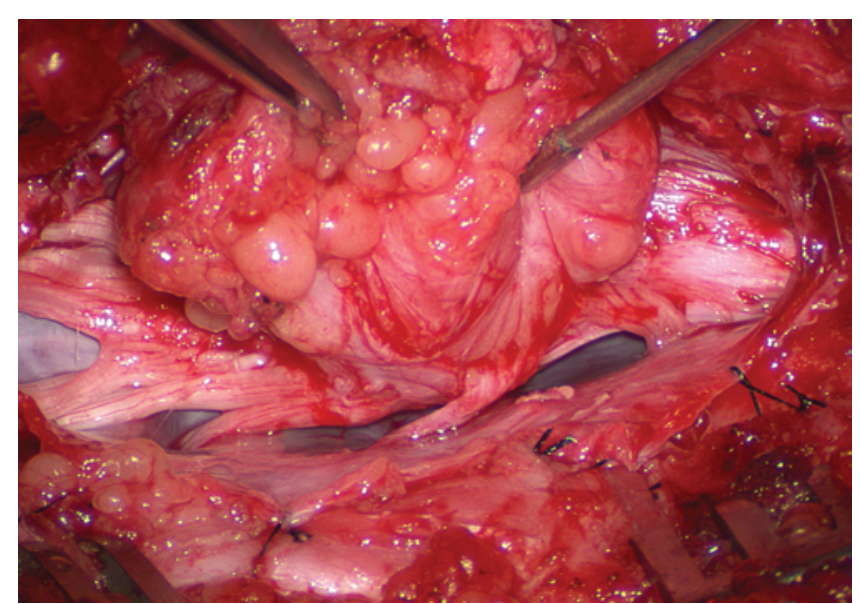

FIG. 6. Crotch dissection is complete on both sides. The entire right array of nerve roots is seen but not the DREZ or the fusion line, which are covered by the lateral overhang of the large lipoma. The entire hammock is now unsuspended from the dura. Reprinted from Pang D: Total resection of complex spinal cord lipomas: how, why, and when to operate. Neurol Med Chir (Tokyo) 55: 695-721, 2015. Published with permission from the Japanese Neurosurgical Society. CC-BY-NC-ND (https:// creativecommons.org/licenses/by-nc-nd/4.0/deed.ja).

first be clearly identified because a good amount of sharp dissection of fat will fall on this line where the lateral edge of the lipoma is attached to the cord just medial to the DREZ. In most dorsal lipomas, the fusion line is traceable as a neat complete oval or circle from side to side, often outlining a flat horizontal plane, and is usually bilaterally symmetrical. This circular line ends short of the conus, which is never involved in the dorsal lipoma (Supplemental Fig. 1B). In a transitional lipoma, the more distinct rostral fusion line resembles that of a dorsal lipoma but distally it becomes blurry and cuts toward the ventral surface of the conus, never joining its mate from the other side at the bottom of the spinal cord (Fig. 3, Supplemental Fig. 2A and B). For a large lipoma of either type, as in the monstrous dorsal lipoma shown in Fig. 6 and Supplemental Fig. 8, exuberant fat may hang over the fusion line and cover up the upper portion of the emergent dorsal roots (the so-called thighs of the roots) to give the false impression that the roots run through and are not separate from the lipoma (Supplemental Fig. 16). In reality, these festoons of overhanging fat can quite easily be sharply dissected off the thighs of the roots to expose the so-called knees of the roots (Supplemental Fig. 17), the DREZ (Supplemental Fig. 18), and the elusive fusion line above (Supplemental Fig. 19). I call this maneuver "knee dissection."

Actual lipoma resection may commence only after the fusion lines from both sides have been clearly identified (Supplemental Fig. 20). Without exception, resection begins at the rostral tip of the lipoma, where the anatomical relationships among fat, nerve roots, and DREZ are clearly decipherable (Supplemental Fig. 21). The first order of business is to use the microscissors to locate a thin but distinct silvery white plane between fat and cord at the demi-lune of the rostral fusion line (Supplemental Fig. 22). The white plane is in essence a thin net of collagen fibers that is distinguishable from yellow fat by its glistening white color and from soft spinal cord by its tough and gritty feel when cut. It can become convoluted and uneven within the main bulk of the lipoma, but its rostral end is almost always distinct and flat (Supplemental Figs. 22C and 23). The initial sharp cut to locate the plane has to be directed seemingly straight into the spinal cord, but after neatly cutting through the tongue of yellow fat at the rostral tip of the lipoma, the gritty white plane can always be safely located here (Supplemental Fig. 24) and, once found, can then be followed by constantly sensing the grittiness through the microscissors and by noting the white glint between yellow globular fat and the dull, pasty pink spinal cord (Supplemental Fig. 25). The $\mathrm{CO}_{2}$ laser should never be used to vaporize the fat because it chars the surface and thus blots out the signature white color of the white plane, and it also casts out the valuable tactile feedback from the microscissors, which unerringly spots the characteristic gritty toughness of the fibrous plane from the softer semiliquid fat. The Nd-YAG laser has a less charring effect on the fat but, without the tactile feedback, is no better in finding or surfing the white plane.

Bleeding on the white plane, which is essentially bleeding on the cord, must be handled delicately. Instead of hastily digging with the sparking bipolar cautery, or worse, scrambling with the suction tip, the exact bleeding spot is accurately and atraumatically located by intermittent gentle jets of irrigation from a small hand bubble-squeezer fitted with a fine blunt needle. Once precisely located by the irrigation, the bleeding can be deftly handled with the ultra-fine irrigating bipolar cautery $(0.2-\mathrm{mm}$ tips, Supplemental Fig. 26) set at a low current. The cold irrigation prevents sticking but more importantly it dissipates heat rapidly from the cord.

While resecting lipoma along the fusion lines (Supplemental Fig. 27), strong diathermy and sharp gouging must be avoided at the DREZ to avert disturbing sensory symptoms. Just medial to the DREZ at the fusion line, a robust cuff of pia mater must be preserved to provide a sturdy stitch-hold for the neurulation sutures (see below) (Supplemental Fig. 23A and B, Fig. 24B). As long as the sharp dissection is kept strictly on the white plane medial to the fusion line and DREZ, complete lipoma resection can be carried out without injury to neural tissues. This is much easier to achieve in most dorsal lipomas because the white plane is usually predictably flat and leveled and because the entire fusion line can be readily charted and circumferentially approached from all $360^{\circ}$ (Supplemental Fig. 28). In a particularly large and rambling dorsal lipoma, the white plane may undulate and ripple considerably so that the corresponding fusion line and its neighboring DREZ will accordingly situate at uneven heights on the side of the cord (Supplemental Fig. 20). Finding this safe zone of dissection may require systematic stimulation of the adjacent nerve roots for functional localization.

Navigating the white plane in most transitional lipomas is considerably more challenging because the plane is never horizontal, usually undulates, and the corresponding fusion line is thus very jagged. One side of the white plane with the DREZ and nerve roots may even be tilted completely away from the surgeon when the entire neural placode is rotated $90^{\circ}$ into the sagittal plane. In such extreme cases, the neural placode now faces one side of the 


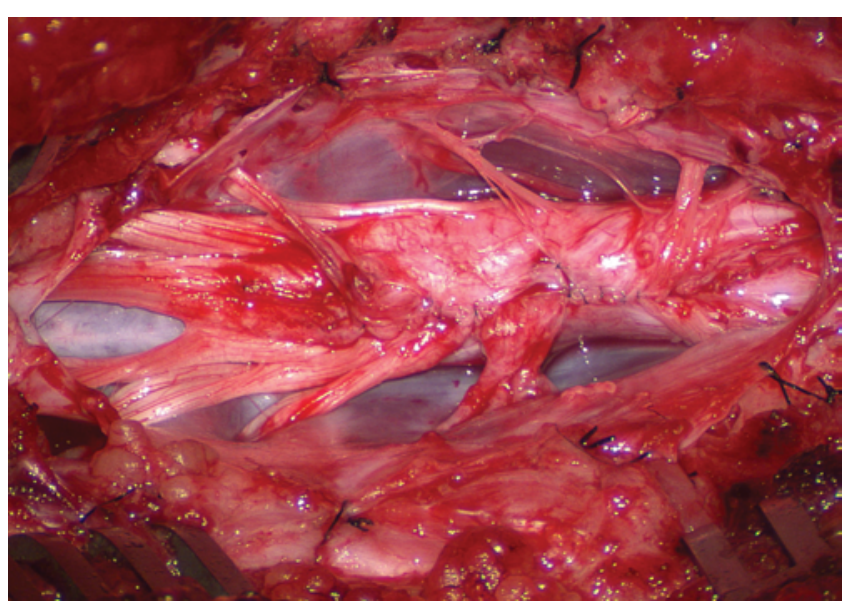

FIG. 7. Pia-to-pia neurulation completed with interrupted 8-0 nylon microsutures. The reconstituted neural tube is entirely pia covered, with an unobtrusive dorsal seam. Note the intact conus. Reprinted from Pang D: Total resection of complex spinal cord lipomas: how, why, and when to operate. Neurol Med Chir (Tokyo) 55: 695-721, 2015. Published with permission from the Japanese Neurosurgical Society. CC-BY-NC-ND (https://creativecommons.org/licenses/by-nc-nd/4.0/deed.ja).

canal and the lipoma the opposite side, and the white plane is now vertical. Not uncommonly, the caudal portion of the lipoma is so large as to obscure all edges of the caudal dural sac, and the white plane dissection feels endless, often amid white bands that could be vestigial or functional nerves. Here, electrophysiological identification of the ventral nerve roots is the only way to determine where functional conus joins the fibrofatty muck of the caudal lipoma. The S2 is the only motor root that activates both the anal sphincter and the abductor hallucis, and the next two sets of puny and adhesion-laden rootlets with "pure" sphincter response must therefore be the S3 and S4 roots. Any suspicious tissue or bands distal to the last "live" sphincter roots can now be considered nonfunctional and be freely discarded to consummate the final emancipation of the neural placode (Supplemental Fig. 29B).

The same principle of using electrical identification of the caudal roots and placode may well be the only way to sort out the true termination of the functional conus in chaotic lipomas. With placode stimulation, the current is increased to 4-6 $\mathrm{mA}$, but the drill is the same as for the motor roots. For the rostrodorsal portion of the chaotic lipoma, dissection of the white plane is as described for dorsal lipomas, but the billows of fat ventral to the placode should be left alone and their pristine pial surfaces left unviolated (Supplemental Fig. 29). It is always the dorsal and never the ventral surface of an untouched lipoma that actually tethers the spinal cord. ${ }^{58}$

For ease of handling, I always try not to cut into the lipoma during the resection because lacerated fat tends to be weepy and fragile and its altered color and texture may confound the dissection front on the white plane. Also, it is more secure to grasp on to firm, encapsulated fat than pulling on dripping, stringy grease. However, for the truly massive lipoma that completely obscures all anatomical landscapes, its central bulk has to be first reduced using the ultrasonic aspirator before its sides can be collapsed inward to locate the crotch hinges.
After the entire lipoma has been resected (Supplemental Fig. 30), it is advisable to systematically stimulate each pair of motor roots on the "naked" placode and to run a few sets of transcortical motor potentials to ensure all baseline functions have been preserved. Functional deterioration has been known to occur during neurulation due to tight strangling of the placode.

\section{Step 4. Pia-to-Pia Neurulation of the Neural Placode}

Thorough resection of fat and fibrous scar converts a stiff, rotund, fat-engorged spinal cord to a supple, slender, and infinitely more maneuverable neural placode suited for tensionless pia-to-pia neurulation (Supplemental Fig. 30). Well-executed neurulation in turn transforms the resection bed from a broad, wafery, sticky flat surface to a single seam on a trim and tidy pia-covered tube, duly minimizing the likelihood of its adhering to the dura.

A few technical tips concerning neurulation deserve special mention. While folding up the neural placode to appose the pial edges, the surgeon may encounter a substantial torque tending to unfurl the folding due to the natural inelastic property of neural tissue. This can be mitigated by applying small Weck clips on the apposed pial cuffs at short intervals along the seam (Supplemental Fig. 31 ), before sewing in the 8-0 nylon pial sutures in between the clips and with buried knots. The Weck clips are then removed after enough sutures have been placed.

Occasionally, strong tugging on the inbending folds prevents their easy coaptation, caused by "short" nerve roots on their underside (Supplemental Fig. 32). These short and presumed to be nonfunctional and "fibrotic" roots have historically been blamed for unsuccessful untethering of the cord. In fact, on electrical stimulation, these roots are often found to be perfectly functional and pliant and have merely been made to appear short and unyielding by stiff adhesion bands to the dura. Cutting these bands releases the roots from the dura (Supplemental Fig. 33A and B) and magically lengthens them (Supplemental Fig. 34) to give a comfortable neurulation (Fig. 7, Supplemental Fig. 35).

I cannot overemphasize the importance of preserving a healthy width of pial cuff on each edge of the placode while resecting fat along the fusion line, not just for providing sturdy stitch-holds for the microsutures, but a generous pial fringe also imparts a certain resilience to the folded structure. Very rarely, the neural placode appears "pressed" into a narrow-based triangle resembling a tall steep pyramid by flanking fat buttresses (Supplemental Fig. 36). The pial cuffs in such cases are also exceedingly narrow, and suturing together short pial cuffs while forcefully folding up a squat, thick pyramid of unyielding neural tissue could result in a strangulated and ischemic heap. The surgeon should recognize the potential hazard of the pyramidal placode on the presurgical MR image and be ready to exercise judgment while reconstructing the placode (Supplemental Fig. 36B-D), which may mean abandoning neurulation, especially when there are adverse changes in the MEPs during infolding and suturing of the placode edges..$^{54,60}$

If a filum is encountered after neurulation, it is usually also resected for completeness. 


\section{Step 5. Expansile Graft Duraplasty}

Countless MR images of loosely floating placodes and the undisputed salutary benefits of a small cord-sac ratio (see below) convinced me that a generous dural graft ensures free movement of the placode in the CSF, thereby lessening its chances of adhering to the dura. The ideal graft material must satisfy two requirements: textural compatibility with juvenile dura and a low incidence of CSF leakage through the suture holes. Gortex is too stiff for young dura and leaks CSF profusely, as does DuraGen. Both materials were discarded in our early trials. Autologous fascia lata is too soft and pliant so that it collapses onto the spinal cord, even during normal inspiration, and is only used when there is an infection negating foreign graft materials. My preferred graft material is the fullbodied yet texturally pliable bovine pericardium (DURAGUARD, Synovis) that can maintain a "puffed-up" scaffold in all phases of respiration and postures and shows minimal CSF leak. A close second choice is Durepair (Medtronic), composed of reconstituted bovine collagen; it is slightly stiffer than bovine pericardium but does not leak. The graft is accurately shaped and sized to the dural opening to avoid creating infolds that may touch on the underlying placode (Supplemental Fig. 37). The edge of the graft is then carefully matched up with the dural opening and anchored with judiciously spaced 4-0 sutures. Final water-tight closure is achieved with several running 5-0 Prolene sutures, followed by several challenges with Valsalva maneuvers using pressures between 20 to $30 \mathrm{~cm} \mathrm{H}_{2} \mathrm{O}$ depending on the patient's size and age (Supplemental Fig. 38). An inadvertent pseudomeningocoele presses the dura against the neural placode and negates any intended perquisite of capacious grafting.

\section{Surgical Epilogue: Sundry Additional Comments}

Our data show that total or near-total resection of spinal cord lipomas can be achieved in over $90 \%$ of cases ${ }^{58-60}$ (Supplemental Fig. 39). In cases in which small bits of fat were left on the DREZ for fear of gouging, they have been wrapped up within the neurulation folds "out of mischief" (Supplemental Fig. 40). In 8\% of patients, the residual fat represents the ventral component of a chaotic lipoma that had been intentionally left untouched and pia covered and presumably harmless.

To estimate the looseness or degree of freedom of the reconstructed placode within the expanded CSF space, we created the cord-sac ratio, defined as the ratio of the diameter of the bulkiest portion of the reconstructed neural placode to the diameter of the dural sac on the postoperative axial MR image. The sacs are graded as loose, with ratios less than $30 \%$; moderately loose, with ratios between $30 \%$ and $50 \%$; and tight, with ratios greater than $50 \%$ (Supplemental Fig. 41). In our series of 315 total resections reported in 2013, $227(72 \%)$ had loose sacs, 73 (23.2\%) had moderately loose sacs, and only 15 (4.8\%) had comparatively tighter sacs..$^{60}$

Redo lipomas are more likely to harbor a visible remainder of fat and to have higher cord-sac ratios. Very likely, the hard, grasping fibrous scar from prior surgery cements the remainder of fat to the dura and makes it extremely difficult to detach. Also, the dull gray color of the cicatrix, unlike the bright yellow virgin fat, can be impossible to distinguish from the white plane. The surgeon thus tends to be over-conservative in the dissection and leaves behind a thick layer of stiff scar-infiltrated fat attached to a bulky placode that is well-nigh impossible to neurulate comfortably. The same unyielding fibrous muddle at the DREZ often leads to "gouging," which may explain the high incidence of postoperative dysesthetic pain in redo versus "virgin" patients. ${ }^{59}$

Incomplete terminal untethering of the placode predictably ends in early clinical relapse. ${ }^{9,61,73}$ We ascribe two explanations for why this could happen with very large transitional lipomas. In such cases in which the caudal placode is mired in fat, its complete detachment cannot be done safely without substantial removal of fat. Also, healthy-looking nerves are sometimes seen enmeshed in fat distal to what was reckoned to be the tip of the placode, making it seem impossible to achieve terminal disconnection without sacrificing functional cord. Electrical stimulation of these nerves will more than likely find them to be nonfunctional, vestigial coccygeal roots, and 2 to 3 sets of "live" anal sphincter roots would have been found on the conus proper above. A decisive transection of the vestigial placode below will achieve complete terminal untethering without sacrificing function. . $^{57,58-60}$

Our experience unequivocally shows that chaotic lipomas are the most treacherous lesions. Some chaotic lesions are easily recognizable by the presence of obvious ventral fat in between pairs of ventral roots on the MRI, but other less blatant ones have subresolution trails of fatty infiltration through the DREZ into the ventral motor horns zone, detectable only at surgery (Supplemental Fig. 29D). Compared to other lipoma types, chaotic lipomas are more likely to show conspicuous residual fat and a high cord-sac ratio on the postoperative MRI. ${ }^{58-60}$ The strategy for these difficult lipomas, gleaned from more than a few infelicitous encounters, is learning to resist digging too deep once enough dorsal fat has been removed to enable partial neurulation. It is worth stressing that since it is never feasible to totally resect the ventral fat or to achieve even clumsy neurulation, its nascent pial surface, which had presumably existed without adhesions since birth, should best be kept unabraded so as not to incur iatrogenic tethering (Supplemental Fig. 29).

Finally, I disagree with Arai et al., ${ }^{1}$ Chapman, and Chapman and Davis ${ }^{8,9,27}$ that lipomyelomeningoceles have higher surgical risk and worse outcome than regular lipomas. As long as one recognizes and gently handles the extruded part of the neural placode beyond the dorsal plane of the spinal canal, the technical steps of fat resection, placode reconstruction, and dural grafting are identical to those used for the more orthodox lipomas, with comparable results. I also think that warnings by Kulkarni et al..$^{27}$ and Chapman ${ }^{8}$ about lipomas in adults being technically more challenging are unwarranted. Except perhaps for thicker "natural" arachnoid bands in adults and the more laborious bone and fascia exposure, most of the microsurgical work is the same as for resecting lipomas in young children and infants. 


\section{Complications of Total Lipoma Resection}

Our rate of new neurological and urological symptoms following total/near-total resection is $4.1 \%,{ }^{60}$ which compares favorably with the $0.6 \%$ to $10 \%$ (average of 3\%-7\%) in the literature, $1,2,10,19,20,21,25,26,29,37,38,41,62,63,69,73$ all pertaining to partial resection presumably using less aggressive techniques than ours. Only $1.5 \%$ of our patients had new weakness, but $4 \%$ had neuropathic pain, ${ }^{58-60}$ which is likely related to mechanical or thermal perturbation at the DREZ and dorsal roots zone from diathermy and gouging. This has taught us to avoid cautery as much as possible and to use gentle tamponade and Gelfoam, which should handle the majority of bleeding on the cord. If diathermy is absolutely necessary, we use only the ultra-fine tip irrigating bipolar set at the lowest current intensities, always precisely localizing the bleeding spot using the intermittent squirt-irrigation technique from the bubble-squeezer.

Our rates for CSF leak $(0.7 \%)$ and wound complications $(1.1 \%)$ with total resection ${ }^{60}$ are much lower than the rates of almost all of the published series (of partial resection), which report CSF leak rates from $2 \%$ to $47 \% \%^{10,19,25,26,29,41,62,69,73}$ and wound dehiscence and infection rates from $2 \%$ to $26 \% .^{19,22,25,29,41,62,69,73}$ The following time-tested technical points concerning wound closure are worth noting: 1) Adequate caudal laminectomy is necessary to locate a healthy dural edge at least $5-10 \mathrm{~mm}$ past the tip of the neural placode to sustain good suture hold for the lower end of the graft anastomosis, which is always the tenuous end. Ideally, this very edge should be sturdy dura and not fat of the distal lipoma stump. A good caudal dural edge is usually available with dorsal lipoma whose fatty stalk ends above the conus as does the dural defect, but in some very large transitional lipomas, the entire conus is involved in the lipoma and the caudal dural edge is partly or wholly infiltrated by fibrous fat. The surgeon will have to improvise on finding the best possible suture hold for the lower end of the graft, occasionally resolving to using "big bites" on fibrous fat or through flakes of the incomplete deep fascia layers. 2) Patience and ingenuity must be used to achieve absolute watertight closure of the graft with Prolene, followed by Valsalva maneuvers. 3) We are not as enchanted with synthetic sealants or organic tissue glues for the graft anastomosis as we once were, for nothing can substitute for solid closure. Use of these agents is optional in cases of tenuous suture line. 4) In large lumbosacral lipomas, gaping muscle and fascia defects may forestall primary approximation of the deep layers at the lower end of the opening. An effective solution is to create generous paramedian relaxing incisions on the flanking lumbodorsal fascia to allow the fascia edges to slide toward the midline and be primarily closed without tension. 5) The large subcutaneous lipoma is never removed during the initial soft-tissue dissection to avoid creating a large dead space that could potentially encourage CSF leakage and formation of a tense pseudomeningocoele. The latter may compress and flatten the dural sac against the placode or even threaten the viability of the skin flap.

\section{Results of Total Resection}

The results of total resection are best analyzed by com- paring its outcome with that of the traditional technique of partial resection. This has proven to be a convenient enterprise for me, who had performed 116 partial resections prior to 1991, using a similar surgical style and similar rituals and with equal obsession for technical minutiae as for the total resection series. These comparisons are done in two parts - the immediate postoperative results and the long-term outcomes-of these two different techniques.

\section{Early Postoperative Results}

The immediate effects of surgery during the first 12 months are very similar between our own total- and partialresection groups.$^{58-60}$ For asymptomatic patients, the rates of neurological preservation are $98 \%$ for total resection and $94 \%$ for partial resection. For symptomatic patients, the rates for total resection are $61 \%$ for normalization or improvement and $33 \%$ for stabilization of neurological status, giving a combined rate of $94 \%$ for improvement or stabilization of disease. Similarly, symptomatic patients who underwent partial resection had a $33 \%$ improvement and $62 \%$ stabilization rates, thus also yielding a $95 \%$ rate of short-term protection.

When the early postoperative results are compared between our total-resection group and partial-resection series from the literature,,$^{1,10,19,29,41,63,73}$ it is again apparent that both the disease improvement and stabilization rates are very similar, particularly among patients with progressive symptoms. This suggests that the immediate benefits of surgery in the early postoperative period, for both techniques, are owed to the abrupt interruption of the tethering effect on the cord rather than the amount of lipoma removal or work on the placode. In contrast, surgeons who lamented their inability to completely detach the terminal placode from the lipoma stump in some patients, due to massive caudal fat and undefinable placode-lipoma anatomy, also reported their early recurrence of symptoms..$^{9,62,73}$

When the catalog of preoperative symptoms is analyzed with regard to their responses to total resection, the abatement of pain is the best and most reliable. Typically, the sharp, dysesthetic pain in the lower limbs and perineum will significantly diminish within 3 months. ${ }^{60}$ Relief of low-back pain, which may be mechanical in origin, is less reliable. ${ }^{18,56}$ Children do not usually complain of back pain, but they do seem to become more active and playful. Active sensory symptoms such as dysesthesia and hyperpathia also respond favorably to surgery. Although only $20 \%$ of patients have actual normalization of motor deficits, the majority with preoperative weakness will substantially improve. ${ }^{58-60}$ Like tethered cord syndrome in general, recent and milder deficits and symptoms have a better chance for good recovery than severe chronic ones. Unlike pain and sensorimotor deficits, bladder dysfunction rarely improves significantly, and if it does improve, the recovery is never complete, and partial recovery occurs in no more than $20 \%-30 \%$ of patients. ${ }^{58,60}$ Among the several subtypes of neuropathic bladder, the spastic, smallcapacity bladder with uninhibited detrusor contractions tends to respond best to surgery. The atonic bladder virtually never improves to a point of the patient not needing permanent intermittent catheterization, and the response 


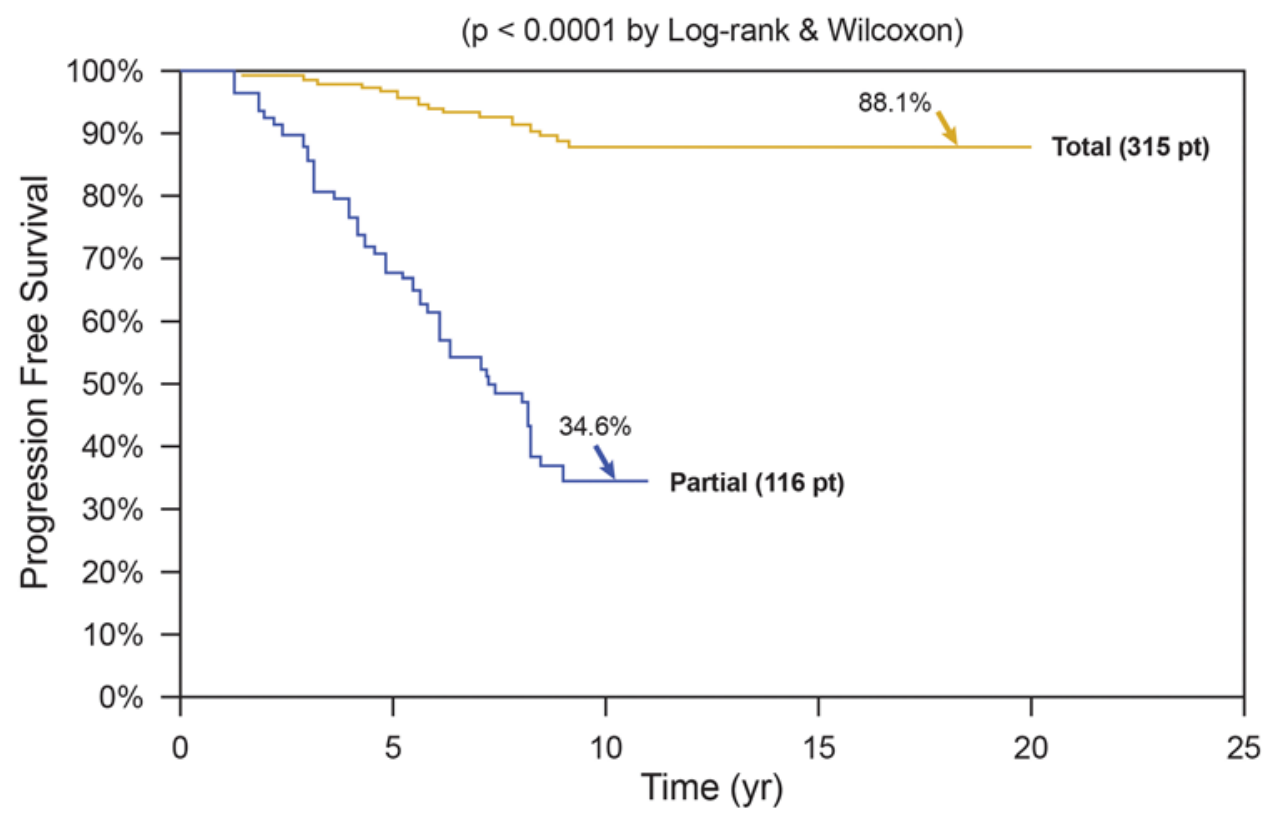

FIG. 8. Outcomes of total versus partial resection. Kaplan-Meier analysis for PFS probability in total and partial resection of lipoma. The progression-free probability for total resection is $88.1 \%$ at 20 years, and $34.6 \%$ for partial resection at 10.5 years. The difference is highly significant $(p<0.0001$ by log-rank and Wilcoxon testing). Note stabilization of disease after 8 years with total resection but inexorable deterioration without disease arrest with partial resection. Partial = partial resection; $\mathrm{Pt}=$ patients; Total = total resection. Reprinted from Pang D: Total resection of complex spinal cord lipomas: how, why, and when to operate. Neurol Med Chir (Tokyo) 55: 695-721, 2015. Published with permission from the Japanese Neurosurgical Society. CC-BY-NC-ND (https://creativecommons.org/licenses/by-nc-nd/4.0/deed.ja).

of detrusor-sphincter dyssynergia is at best partial. It is strongly advisable to obtain formal cystometry testing and a voiding cystourethrogram 3-6 months after surgery and yearly thereafter, if indicated, to assess whether more radical urological procedures, such as bladder augmentation or ureteral conduits, might be needed to eliminate reflux and upper urinary tract infections.

\section{Long-Term Outcome}

While the immediate benefits of both total and partial resection are due to the equivalent relief of traction on the conus in both procedures and are therefore comparable, ${ }^{58-60}$ the long-term benefits of total over partial surgery probably come from the sustainment of the untethered state when the lipomatous cord underwent aggressive fat removal and protective reconfiguring of the neural structure, an undertaking obviously unobtainable with partial resection. This is made very plain when the progression-free survivals (PFSs) of our own series of total and partial resections are plotted out for periods of 20 and 11 years, respectively. The PFS rates after total resection for all lipoma types and clinical subgroups is $88.1 \%$, versus $34.6 \%$ for partial resection ${ }^{60}$ (Fig. 8). The differences in outcome between the two techniques are even more marked if the subgroup PFSs are individually compared, when the patients in each treatment group are segregated by the presence or absence of preoperative symptoms, age, and whether previous surgery had been done ${ }^{58-60}$ Overall, the risk of symptomatic recurrence is 5.94 times higher with partial resection than with total/near-total resection over 18 years, according to the Cox proportional hazards regression model $(\mathrm{p}<0.00001)$. This striking difference in outcomes between total and partial resection is equally obvious when the results of our total resection group are held up against the published results of partial resection, $1,8,11,12,41,62,63,66,67,69,73$ especially if the comparisons are done with matched patient profiles (most published series include only virgin cases, whereas ours has both virgin and redo cases) and lesion types (many published series include terminal and filar lipomas, which are technically far less complicated and have a better prognosis). Barring these and other minor criticisms (e.g., our data are prospective, whereas most published series are retrospective), these elaborate comparisons all show a far better longterm prognosis with total/near-total resection, which constitutes the best reason for its endorsement over all other treatment options for complex spinal cord lipomas.

\section{Predictor Variables Influencing Outcome}

In 2010, we attempted to find the actual reason(s) why total resection yielded such a stunning advantage over partial surgery, by investigating the influence of 6 predictor variables on PFS in the series..$^{59}$ These variables are sex, age, lipoma type (dorsal, transitional, or chaotic), presence or absence of preoperative symptoms, history of previous resection, and cord-sac ratio. Simple Cox univariate analyses showed that sex and lipoma type had no influence on outcome but that young age, absence of symptoms, no prior surgery, and a low cord-sac ratio are all associated with statistically longer PFS. However, when age, symptoms, and prior surgery (not cord-sac ratio because it is a postoperative entity) are tested with the Cox multivariate model with all 6 variables inserted in the analysis, the significant $p$ 
( $p<0.0001$ by Log-rank \& Wilcoxon between Total and Partial)

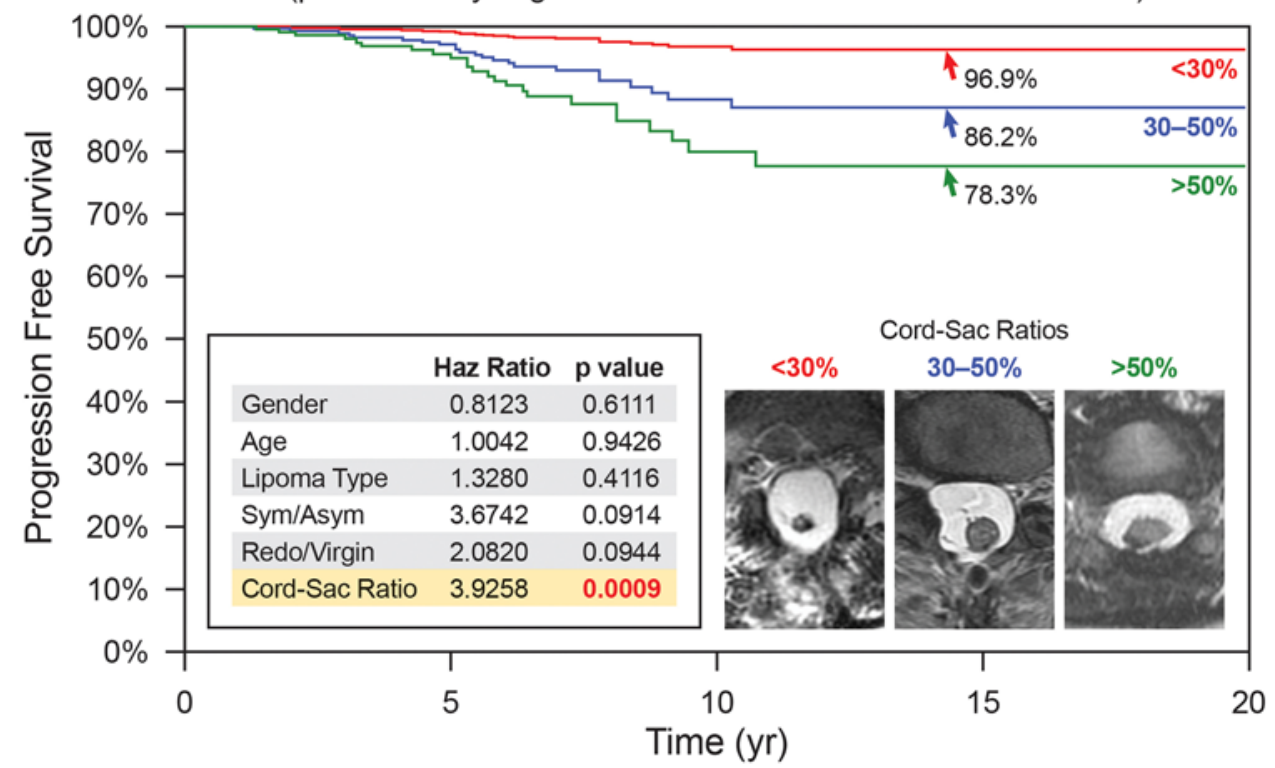

FIG. 9. Cox multivariate proportional hazard regression model analyzing the combined influence of 6 predictor variables (sex [gender]; age; lipoma type; symptoms; redo versus virgin lipoma; and cord-sac ratio) on PFS after total resection, featuring the resultant effect of the 3 cord-sac ratios of $<30 \%, 30 \%-50 \%$, and $>50 \%$. The hazard ratios (Haz Ratio) and $p$ values for all 6 predictor variables are listed in the miniaturized table, showing that cord-sac ratio exerts the only significant independent influence on outcome. The respective progression-free probabilities, as indicated by the arrows, are $96.9 \%$ for a low ratio, $86.2 \%$ for an intermediate ratio, and $78.3 \%$ for a high ratio. The differences in hazard prediction for the 3 ratios are highly significant $(p=0.0009$, in bold). $<30 \%, 30-50 \%$, and $>50 \%$ indicate the 3 cord-sac ratios as shown in MRI insets. Sym/Asym = symptomatic versus asymptomatic lipomas. Reprinted from Pang D: Total resection of complex spinal cord lipomas: how, why, and when to operate. Neurol Med Chir (Tokyo) 55: 695-721, 2015. Published with permission from the Japanese Neurosurgical Society. CC-BY-NC-ND (https://creativecommons.org/licenses/by-nc-nd/4.0/deed.ja).

values associated with their univariate PFS curves all disappeared, suggesting these 3 are in fact non-independent predictors of outcome. ${ }^{59}$ The same statistical exercise was performed in our 2013 series of 315 patients, with similar results. ${ }^{60}$ The inevitable conclusion is that these 3 predictor variables must exert their influence on outcome through some sort of final common pathway. ${ }^{58-60}$

Unlike the 3 non-independent predictor variables of age, symptoms, and prior surgery, cord-sac ratio emerges as the solitary independent predictor of outcome shown clearly by its highly significant influence on progression-free probabilities displayed by the Cox multivariate model $^{60}$ (Fig. 9). It is therefore tempting to suggest that the cord-sac ratio may in fact be the final common pathway for the three non-independent predictors. To test this hypothesis on the influence of prior surgery on outcome, we examined whether redo lipomas' negative effects on PFS can be explained solely by their having high cordsac ratios. The first positive clue is that redo lipomas tend to end up with much higher postoperative cord-sac ratios than virgin lipomas. ${ }^{58-60}$ Second, ANCOVA clearly portrays the same strong correlation between prior surgery and high cord-sac ratios $\left(\mathrm{R}^{2}\right.$ correlative coefficient $=0.76$; $\mathrm{p}<0.001) .{ }^{60}$ Last and most revealing, when the Cox multivariate regression analysis was performed on cord-sac ratio for just the redo lipomas, a PFS of $88.4 \%$ was still manageable in redo lesions as long as a less than $30 \%$ cord-sac ratio can be rendered ${ }^{60}$ (Supplemental Fig. 42). This proves that redo lesions can still enjoy good outcome as long as a low cord-sac ratio can be achieved, and clearly shows the subordinate role of other unidentified attributes of prior surgery. This same train of analyses, including the respective ANCOVA, can be applied to the other two nonindependent predictors, age and symptoms, with similar conclusions.

\section{Cord-Sac Ratio and the Importance of Neurulation}

My own records show a far higher likelihood for total resection to achieve low cord-sac ratios than partial resection: $72 \%$ in the total group and only $3 \%$ in the partial group (Supplemental Fig. 43), suggesting that cord-sac ratio is probably what determines the difference in outcome between the two techniques.$^{60}$ For the total resection group, Cox multivariate analysis of the combined influence of the 6 predictor covariates shows that cord-sac ratio's highly significant, independent influence on outcome is unchanged by however the other effects of age, symptoms, lipoma types, and prior surgery interact (Fig. 9). Regardless of all else, a cord-sac ratio greater than $50 \%$ predicts a 5.3-times-higher risk for disease progression than a low cord-sac ratio of less than $30 \%$, with a high statistical significance (Figs. 9 and 10). The PFS rises to a reassuring $96.9 \%$ for low ratios less than $30 \%$, drops gradually to 

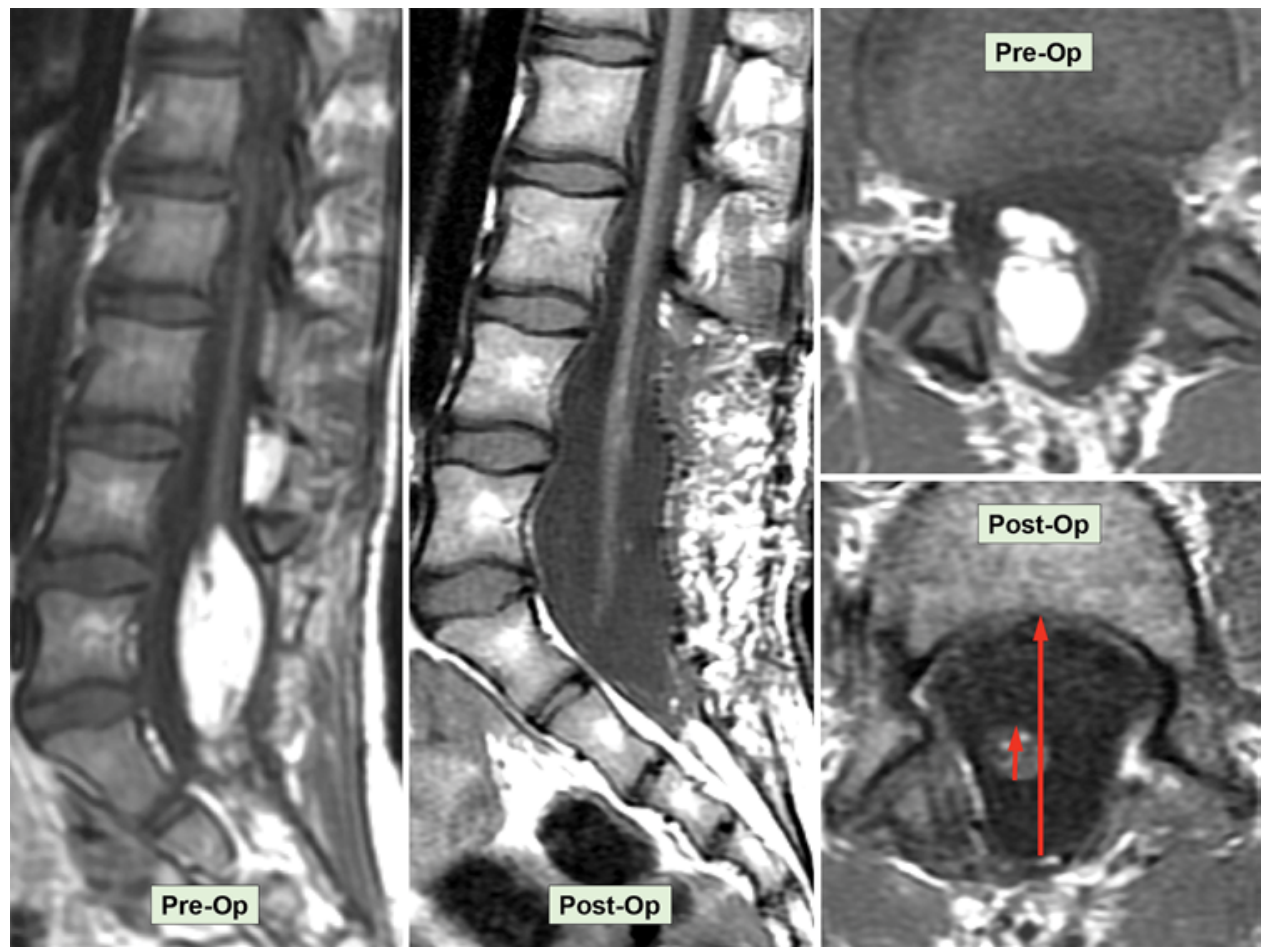

FIG. 10. Pre- and postoperative sagittal (left) and axial (right) MR images of a 10-year-old girl, who has had 2 previous partial resections of a large transitional lipoma and who developed new leg weakness. The achieved postoperative cord-sac ratio is $20 \%$ (lower right); and she enjoyed long-term progression-free status. Arrows point to "free conus" completely uninvolved with the lipoma. Reprinted from Pang D: Total resection of complex spinal cord lipomas: how, why, and when to operate. Neurol Med Chir (Tokyo) 55 : 695-721, 2015. Published with permission from the Japanese Neurosurgical Society. CC-BY-NC-ND (https://creativecommons.org/ licenses/by-nc-nd/4.0/deed.ja).

$86.2 \%$ for intermediate ratios between $30 \%$ and $50 \%$, and is $78.3 \%$ for ratios higher than $50 \% .^{60}$

In an operational sense, the cord-sac ratio may in fact be the summated product of the other group variables. For example, previous surgery imparts new scar tissue on the remainder fat, whose altered hue and texture undoubtedly make it nearly impossible to separate lipoma from white plane and therefore to attain a clean, slender placode and a small cord-sac ratio. Since many symptomatic patients in our total resection group have undergone prior partial resection, their poorer outcome may be largely due to the negative influence of the "redo factor," which may also account for the disadvantage of old age since most elderly patients had both symptoms and prior surgery before their total resection.

Though a loose-fitting sac ensures a greater degree of free movements of the placode within the CSF, a condition known to discourage retethering, adhesions can still form if what is facing the dura is a broad, sticky, raw resection surface on a sheet-like placode. Meticulous pia-topia suturing conceals this sticky surface within a smooth pia-covered tube and further lessens the chances of adhesion. Reexploration of both neurulated and unneurulated placodes shows unequivocally that the raw unneurulated slab is much more of a conspicuous nidus for new adhesion bands than a smooth tube with a discrete seam. Though not an easily quantifiable act, concealment of this adhesive surface through minutely careful neurulation must be at least as essential and indispensable as a low cord-sac ratio in guarding against retethering.

\section{Preoperative Profiling for Good and Poor Risk Patients}

In clinical practice, it is important to inform patients and families of their chances of success before the recommended treatment, and for that, the postoperative index of cord-sac ratio, though by far the most potent outcome predictor, will not be available. To create a presurgery composite patient profile to differentiate potentially favorable from unfavorable surgical candidates, the strengths of influence on outcome of all preoperative traits derived from the predictor variables are ranked on a graded map from another multivariate data analysis model, multiple correspondence analysis (MCA). In an MCA 3D map, the individual strength of influence on outcome of each of the 5 preoperative predictor variables-age, sex, lipoma type, symptoms, and prior surgery-is graded so that its magnitude is inversely proportional to its distance from a pertinent outcome category, displayed on a "principal component axis" on the map. A short distance from the outcome category indicates strong influence, and conversely, a long distance indicates minimal influence. Thus, at a glance, the traits clustering closest to the outcome category on the MCA map would have a dominant influence, and, vice versa, those traits far afield would have little or no influence. Outcome is considered "bad" for those who had a postoperative recurrence of symptoms and "good" for those 
who had no recurrence documented during the follow-up period. ${ }^{58-60}$

The MCA map shows that bad outcome is strongly associated with lipomas in patients with symptoms and prior resection (Supplemental Fig. 44). Good outcome is, not unexpectedly, correlated with young age, especially less than 2 years, absence of symptoms, and no prior surgery (Supplemental Fig. 45). Capitalizing on the MCA results, we performed a Cox regression analysis on various patient groups that had total resection and found that the PFS probability in the 84 patients who fit this ideal profilei.e., asymptomatic children less than 2 years with no previous resection-maintains at a stunning $99.2 \%$ at 20 years (Supplemental Fig. 46). The ideal scenario with the highest probability of obtaining long-term disease control is therefore to perform total resection on very young children before symptoms arise and most definitely before partial resection has been inflicted. Indeed, our subgroup of 139 patients who had their virgin lipomas resected when young all had postoperative cord-sac ratios $<30 \%$, and none had recurrence during the 20-year follow-up period.

In stark contrast, older patients with preoperative neurological dysfunctions and a history of surgery will have a much less sanguine future.

\section{Total Resection Versus No Surgery for Asymptomatic Lipomas}

Above all, the most important and certainly most often asked question is undoubtedly whether surgery should be offered to children with asymptomatic lipomas. In 2004, a cohort of 50 children with asymptomatic lipomas were prospectively followed without surgery for 9 years at L'hôpital Necker-Enfants malades, Paris, ${ }^{27}$ and were found to have a deterioration risk of $33 \%$ with a progression-free survival probability of $67 \% .{ }^{27}$ A retrospective study from Great Ormond Street Hospital, London, with similar patient numbers ( 56 children) and profiles also reported a 10 -year disease progression rate of $40 \%$ and PFS of only $60 \%$ with nonsurgical treatment. ${ }^{72}$ The London study also found that female sex, transitional lipomas, and conus syringes are all associated with a higher rate of deterioration, which also occurs earlier than patients not having these traits. Since transitional lesions and females are usually more numerous in most lipoma cohorts, as are conus syringes, one could surmise that there is a large subclass of children with these traits who will have a worse than $40 \%$ prospect of disease progression $^{48}$ (Supplemental Fig. 47). Given that children within the lipoma age range have an actuarial life span of 65 to 70 years, and that established neurogenic bladder and motor weakness seldom fully recover following post factum salvage surgery, $2,6,29,41,69,73$ the push for preemptive surgery for asymptomatic lipomas would seem logical if it can be shown to offer clear-cut outcome advantage over nonsurgical management.

Total resection, as is amply shown here, does just that. When the subgroup of young asymptomatic children with virgin lipoma from our total resection cohort is put up against the Paris and London groups, the superiority of total resection is obvious, with a 20 -year PFS of $98.8 \%^{60}$ compared to the 10 -year rates of $67 \%$ and $60 \%$ for conser- vative management in Paris and London, respectively ${ }^{27,72}$ (Fig. 11). In addition, our total resection PFS curve shows disease stabilization 5 years after surgery, whereas there is probably continued disease progression beyond 9 or 10 years with no surgery. ${ }^{27,72}$ There is thus strong statistical support for endorsing total resection, not only for symptomatic children but also as prophylactic treatment for asymptomatic children with lipomas, at least those with the dorsal and transitional types (see below for chaotic lipomas).

There are currently no data on the rate or probability of disease progression for adults with asymptomatic lipomas, and their actuarial life span is obviously shorter than that of children, so the cumulative risk for adults should be far smaller. A forceful argument therefore cannot be made for prophylactic surgery in asymptomatic adults, but when a lipoma becomes symptomatic, further deterioration is usually inevitable, and aggressive resection can then be justified for both children and adults.

\section{Is Partial Resection Worse Than No Resection?}

I would very much like to register my definitive answer to this question and hopefully put the issue of partial resection to rest. To start, the 11-year PFS probability of 34.6\% from our own partial-resection series of 116 lipomas $^{58-60}$ is clearly inferior to the Paris (PFS of 67\%) and London (PFS of 60\%) series ${ }^{27,72}$ of observation alone. If we now calculate outcome only in our asymptomatic virgin patients who underwent partial resection to match the profiles of the other two series, in essence eliminating the fastidious redo lipomas, the 11-year PFS of these patients after partial surgery is only 43\%,59 still far short of the Paris and London numbers. ${ }^{27,60,72}$ Moreover, the Paris series of partial resection ${ }^{27}$ managed only a slightly higher PFS of $51 \%$ (Fig. 11), and other similar series reported equally dismal or worse PFSs, far worse than no surgery. Minus the goodrisk terminal lipomas, Colak and associates' series ${ }^{11}$ would have a less than a 50\% PFS, as would the partial resection series of Pierre-Kahn et al. (< 40\% PFS) ${ }^{62}$ Cochrane and colleagues' series ${ }^{10}$ of partial resections of transitional lipomas had a 10 -year PFS of only $20 \%$, and Cornette et al. ${ }^{12}$ conceded unaltered progression of disease after partial resection.

The explanation for the aforementioned statistics is quite plain if one had the unpleasant experience of exploring these redo lipomas, as I did more than 150 times and witnessed how the abraded but unneurulated placode could be solidly welded to the dura by rigid scar much more than in an unoperated lipoma with its nascent surface. If the frequency and speed of symptomatic recurrence are proportional to the extent and firmness of the fixity of the placode, one would certainly expect partial resection to incur sooner and more severe functional impairment than if no surgery had been done, which is clearly shown in our 2013 analysis. ${ }^{60}$ The time course of in situ scar formation also explains why partial resection typically yields immediate clinical improvement owing to the initial relief of tethering but is unable to sustain these short-lived benefits against the slow but inexorable course of chronic scar formation, 


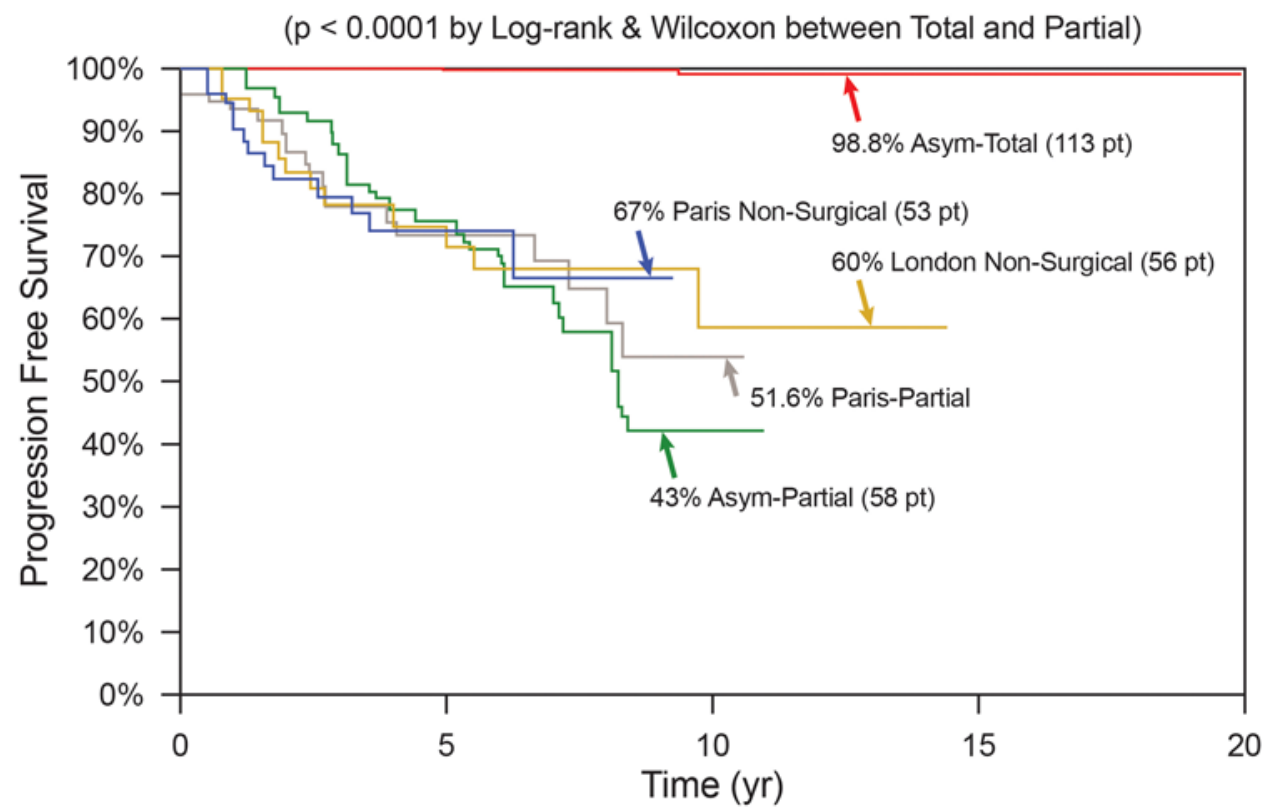

FIG. 11. Outcome differences among total resection, partial resection, and nonsurgical management of asymptomatic virgin lipomas by Kaplan-Meier analysis. The nonsurgical PFS graphs ("Paris Non-Surgical") from the Parisian study ${ }^{66}$ and from the London study ${ }^{68}$ ("London Non-Surgical") are inserted for visual comparison only and not meant to imply a true "head-to-head" comparison. PFS probability at 20 years for the 113 asymptomatic virgin lipomas that had undergone total resection is $98.8 \%$, much better than $67 \%$ of the Paris series or the $60 \%$ of the London series and far superior to the $43.3 \%$ of our own partial-resection series and the $51.6 \%$ of the partial-resection series in the Paris study. The difference between total and partial resection for asymptomatic virgin lipomas is highly significant $(p<0.0001)$. Asym-Partial = asymptomatic virgin lipomas treated by partial resection; Asym-Total = asymptomatic virgin lipomas treated by total resection; London Non-Surgical = asymptomatic virgin lipomas from London; Paris Non-Surgical = asymptomatic virgin lipomas managed nonsurgically in Paris. The Paris partial-resection (Paris-Partial) and our own partial-resection series (Asym-Partial) are as labeled. Reprinted from Pang D: Total resection of complex spinal cord lipomas: how, why, and when to operate. Neurol Med Chir (Tokyo) 55: 695-721, 2015. Published with permission from the Japanese Neurosurgical Society. CC-BY-NC-ND (https://creativecommons.org/licenses/by-nc-nd/4.0/deed.ja).

ending in an unrelenting downhill path that is clearly worse than the natural history of the disease.

Our extensive literature search suggests that the current global practice of lipoma surgery comprises a continuum of techniques ranging from perfunctory whittling of fat with no chance of placode refurbishment to aggressive total resection of lipoma, elaborate microsurgical reconstruction of the placode, and capacious dural grafting, as is endorsed here. In between these two extremes, there is at least one historical series that describes the half-measured protocol of "subtotal fat removal" and "partial neurulation" and reports somewhat better results than nonsurgical treatment. ${ }^{29}$ It is thus tempting to hypothesize that the thoroughness of "clean" placode reconstruction, which is estimable by MRI, is commensurate with the clinical benefits and that the projected outcome of nonsurgical treatment lies somewhere between the two extremes of skimpy and complete fat removal. This would also imply that partial resection will in the long run prove more harmful than no surgery for the patient. In this context, I rather strongly think that partial resection should be discouraged.

\section{Unsettled Issues}

\section{Lipoma Types and Outcome and the Dubious Rank of Chaotic Lipomas}

According to our total resection data, the type of lipoma should not affect outcome projection,,$^{59,60}$ but in reality it probably does. One of the limitations of biostatistics is its inherent fallacy of rigidly assigning finite variables to represent the almost infinite vicissitudes of biological forms. For example, under the same umbrella of transitional lipomas, there are lesions with relatively orderly alignment of the DREZ and nerve roots, a predictable lipoma-cord interface, and manageable quantities of fat, and there are also lipomas with plumelike billows of smothering fat that defy designation. The success and complication rates for these two extremes cannot be the same, and indiscriminately blending their divergent statistical weights is accordingly misleading. Thus, the blanket statement of "no statistical difference" in outcome between lipoma types should not be taken too literally, nor should it supplant an experienced surgeon's impression, despite comforting statistics, that some sprawling, intractable transitional lipomas demand much more exacting skill and portend a graver prognosis than a tamer, friendlier dorsal lipoma.

By far the most difficult and treacherous lipomas I have ever resected are of the chaotic type. The demarcation between fat and neural tissue is sometimes indiscernible even within the dorsal portion of these lesions, and the resection should be more conservative and aim only to remove just enough dorsal fat to enable a comfortable neurulation of the dorsal part. I have repeatedly emphasized restraint and avoidance of doing damage to the pia covering the ventral fat since its complete resection and ventral neurulation are never possible. Consequently, it is not uncommon for 
chaotic lipomas to have higher cord-sac ratios and display more residual fat than the other two lipoma types on the postoperative MRI (Supplemental Fig. 29D).

When in 2010 we compared the total resection outcomes of the 3 lipoma types in a cohort of 238 patients, we found no actual statistical differences between them. ${ }^{59}$ However, even with the caveat of small sample size of only 10 chaotic lesions, the PFS curve for chaotic lipomas ran considerably lower than the curves for dorsal and transitional lesions, and the $\mathrm{p}$ values between the chaotic and the other two lesions were only very slightly higher than 0.05 , giving us the preliminary suspicion that chaotic lipomas had a worse prognosis than the others. In our more recent series of 315 total resections published in 2013, ${ }^{60}$ the number of chaotic lipomas had risen to 28 . The PFS rates for dorsal and transitional lipomas of $92.2 \%$ and $86 \%$, respectively, remained very similar to the 2010 figures, and again they had clearly no statistical difference, with $\mathrm{p}=0.458$. However, the PFS rate for chaotic lesions had dropped slightly to $54 \%$ and its regression curve separated even further away from the other two curves than in 2010 (Supplemental Fig. 48). Within this chaotic group, there were no patients with cord-sac ratios lower than $30 \%$, and their postoperative MR images often showed a hefty placode with a loathsome amount of remainder ventral fat and a tight container fit. ${ }^{60} \mathrm{By}$ strict standards, the outcome differences between chaotic and the other 2 lipoma types only barely reached statistical significance ( $p=0.0492$ for dorsal lipomas; $p=0.0481$ for transitional lipomas), but given larger numbers in the future, the rank of chaotic lesions might very well be definitively downgraded to the most sinister of the three lipoma types.

In Supplemental Fig. 49, I inserted the London outcome curve of nonsurgical treatment onto the PFS rates of the 3 lipoma types. The 54\% PFS of chaotic lipomas, after aggressive resection, is actually worse than the rates of both the London and Paris series of nonsurgical treatment, which begs the question whether asymptomatic chaotic lipomas should be treated with any prophylactic surgery, regardless of technique. This sobering observation has changed my 2010 stance of prophylactically resecting all types of asymptomatic lipomas, and I now recommend that asymptomatic chaotic lesions, if unmistakably diagnosed on MRI, should be closely observed without surgery until symptoms develop.

\section{Asymptomatic Versus Symptomatic Lipomas}

For all the lofty statistics and extravagant analyses, there remains the unanswered question whether symptomatic lipomas do worse than asymptomatic ones after total resection. In our 2013 series of 315 patients, ${ }^{60}$ multivariate analysis found no statistical difference in outcome between symptomatic and asymptomatic lesions, but univariate analysis, in contrast, showed that asymptomatic lesions had significantly better outcome than symptomatic lesions, as we had found in our earlier series of 238 patients (Supplemental Fig. 50). Even when the analysis is run just on the virgin lesions to eliminate the redo factor among symptomatic patients, asymptomatic patients still came out much better ${ }^{60}$ (Supplemental Fig. 51).

In addition to our own results, several large series in the literature ${ }^{16,29,62}$ have also reported considerably better outcome for asymptomatic than symptomatic patients. It was once argued that children with symptoms tend to be older and are thus more likely to undergo growth spurts and take up rigorous sports, both situations subjecting the spinal cord to pernicious tugging known to trigger symptoms in other tethered cord conditions, whereas asymptomatic children are much younger. The post-resection profiles of the two groups are thus not comparable, as the symptomatic older child is being followed at a more vulnerable age..$^{29}$ While this may explain some cases of early recurrence in the symptomatic group, Byrne et al., in a series of 100 infants that eliminated the age difference in the two groups, still showed a markedly better outcome for asymptomatic infants. ${ }^{6}$ Current consensus seems to support the notion that there are indeed prognostic differences between symptomatic and asymptomatic lipomas.

To date, no consistent dissimilarity in size, architecture, or histology has been found between symptomatic and asymptomatic lipomas, but there may be invisible differences in susceptibility inherent within their respective spinal cords. Perhaps the already injured cord that is showing symptoms has become more "fragile" and possesses a lower threshold for new symptoms with even slight retethering. Perhaps those spinal cords, which develop early symptoms, are also intrinsically predisposed to sustain new injuries to even minor future insults and are thus destined to have earlier disease recurrence.

\section{Conclusions}

Total/near-total resection of complex spinal cord lipomas and complete reconstruction of the neural placode produce a much better long-term symptom-free survival than partial resection. There is, in fact, incontrovertible evidence that partial resection causes new and exuberant scarring between the cut surface of the remainder fat and the dura, resulting in earlier and firmer retethering and consequently a worse prognosis compared to no surgery. Our postoperative neuro-urological and wound complications for total resection are either comparable to, or lower than, most series of partial resection.

In matching our results of total resection to published results of nonsurgical management of lipomas, we found that total resection confers much greater long-term benefits than no surgery in children with asymptomatic virgin lipomas. The ideal patient profile for total resection predictive of early disease stabilization and the longest recurrencefree survival has been identified to be a child less than 2 years of age who is without symptoms or a history of surgery. We therefore strongly recommend prophylactic total resection for asymptomatic dorsal and transitional lipomas in children. Multivariate analysis have also shown that a low postoperative cord-sac ratio is highly predictive of good long-term outcome. My firm impression is that a well-executed neurulation is just as important in preventing retethering.

Our experience so far shows that chaotic lipomas are the most treacherous of the 3 types, and the majority are simply not amenable to thorough resection or optimal placode reconstruction, let alone attaining the goal of a low 
cord-sac ratio. Their poor statistics are unlikely to improve, even with larger numbers, and consequently I do not recommend prophylactic surgery for asymptomatic chaotic lipomas.

A lengthy analysis like this one is futile unless it can provide at least a few serviceable truths, perhaps as succinct answers to the 3 questions asked in the title of this review. The "how" of total resection, i.e., its technical aspects, can be learned by any willing neurosurgeon with a modicum of patience, tenacity, and open-mindedness. Those who have observed the surgery will attest that other than a few unaccustomed maneuvers and tricks, which can be learned, the rest is strictly standard microsurgery. The intraoperative electrophysiology work can be managed by any trained neurophysiologist, and the equipment should be purchasable at reasonable cost.

As to the "why" and "when" questions, our massive outcome data collected over 25 years should have given eloquent reasons to endorse total resection for asymptomatic dorsal and transitional lipomas in children and for all symptomatic lipomas in all ages. The surgery should be done soon after diagnosis, except in very young infants with stable neurology, for whom surgery is delayed till 6-12 months of age to minimize surgical and anesthetic morbidity. Surgery for asymptomatic chaotic lipomas is withheld until symptoms develop.

Last, to conclude the conclusion, I should add that "seeing is believing," and anyone wishing to witness our technique first-hand, appended with personal tutelage, is welcomed to contact us to make arrangements.

\section{References}

1. Arai H, Sato K, Wachi A: Surgical management in 81 patients with congenital intraspinal lipoma. Childs Nerv Syst 8:171, 1992

2. Atala A, Bauer SB, Dyro FM, Shefner J, Shillito J, Sathi S, et al: Bladder functional changes resulting from lipomyelomeningocele repair. J Urol 148:592-594, 1992

3. Barson AJ: The vertebral level of termination of the spinal cord during normal and abnormal development. J Anat 106:489-497, 1970

4. Bruce DA, Schut L: Spinal lipomas in infancy and childhood. Childs Brain 5:192-203, 1979

5. Brunelle F, Sebag G, Baraton J, Carteret M, Martinat P, Pierre-Kahn A: Lumbar spinal cord motion measurement with phase-contrast MR imaging in normal children and in children with spinal lipomas. Pediatr Radiol 26:265-270, 1996

6. Byrne RW, Hayes EA, George TM, McLone DG: Operative resection of 100 spinal lipomas in infants less than 1 year of age. Pediatr Neurosurg 23:182-187, 1995

7. Caldarelli M, McLone DG, Colins JA, Suwa J, Knepper PA: Vitamin A induced neural tube defects in a mouse. Concepts Pediatr Neurosurg 6:161-171, 1985

8. Chapman PH: Congenital intraspinal lipomas: anatomic considerations and surgical treatment. Childs Brain 9:37-47, 1982

9. Chapman PH, Davis KR: Surgical treatment of spinal lipomas in childhood. Pediatr Neurosurg 19:267-275, 1993

10. Cochrane DD, Finley C, Kestle J, Steinbok P: The patterns of late deterioration in patients with transitional lipomyelomeningocele. Eur J Pediatr Surg 10 (Suppl 1):13-17, 2000

11. Colak A, Pollack IF, Albright AL: Recurrent tethering: a common long-term problem after lipomyelomeningocele repair. Pediatr Neurosurg 29:184-190, 1998

12. Cornette L, Verpoorten C, Lagae L, Plets C, Van Calenbergh F, Casaer P: Closed spinal dysraphism: a review on diagnosis and treatment in infancy. Eur J Paediatr Neurol 2:179-185, 1998

13. Detwiler SR, Holtzer H: The inductive and formative influence of the spinal cord upon the vertebral column. Bull Hosp Jt Dis 15:114-123, 1954

14. Dias M, Pang D: Human neural embryogenesis: a description of neural morphogenesis and a review of embryonic mechanisms, in Pang D (ed): Disorders of the Pediatric Spine. New York: Raven Press, 1994, pp 1-26

15. Dick EA, de Bruyn R: Ultrasound of the spinal cord in children: its role. Eur Radiol 13:552-562, 2003

16. Dorward NL, Scatliff JH, Hayward RD: Congenital lumbosacral lipomas: pitfalls in analysing the results of prophylactic surgery. Childs Nerv Syst 18:326-332, 2002

17. Hamilton HL, Boyd JD, Mossman HM: Human Embryology, ed 4. Baltimore: Williams \& Wilkins, 1972

18. Hoffman HJ, Hendrick EB, Humphreys RP: The tethered spinal cord: its protean manifestations, diagnosis and surgical correction. Childs Brain 2:145-155, 1976

19. Hoffman HJ, Taecholarn C, Hendrick EB, Humphreys RP: Management of lipomyelomeningoceles. Experience at the Hospital for Sick Children, Toronto. J Neurosurg 62:1-8, 1985

20. James CCM, Lassman LP: Diastematomyelia and the tight filum terminale. J Neurol Sci 10:193-196, 1970

21. James HE, Canty TG: Human tails and associated spinal anomalies. Clin Pediatr (Phila) 34:286-288, 1995

22. James HE, Williams J, Brock W, Kaplan GW, U HS: Radical removal of lipomas of the conus and cauda equina with laser microneurosurgery. Neurosurgery 15:340-343, 1984

23. Jones PH, Love JG: Tight filum terminale. AMA Arch Surg 73:556-566, 1956

24. Källén B: Early embryogenesis of the central nervous system with special reference to closure defects. Dev Med Child Neurol 19:16, 44, 1968

25. Kanev PM, Lemire RJ, Loeser JD, Berger MS: Management and long-term follow-up review of children with lipomyelomeningocele, 1952-1987. J Neurosurg 73:48-52, 1990

26. Koyanagi I, Iwasaki Y, Hida K, Abe H, Isu T, Akino M: Surgical treatment supposed natural history of the tethered cord with occult spinal dysraphism. Childs Nerv Syst 13:268274, 1997

27. Kulkarni AV, Pierre-Kahn A, Zerah M: Conservative management of asymptomatic spinal lipomas of the conus. Neurosurgery 54:868-875, 2004

28. Kunitomo K: The development and reduction of the tail and of the caudal end of the spinal cord. Contrib Embryol 8:161-198, 1918

29. La Marca F, Grant JA, Tomita T, McLone DG: Spinal lipomas in children: outcome of 270 procedures. Pediatr Neurosurg 26:8-16, 1997

30. Marin-Padilla M: Clinical and experimental rachischisis, in Vinken PS, Bruyn GW (eds): Handbook of Clinical Neurology. Amsterdam: North-Holland, 1978, Vol 32, pp 159-191

31. Marin-Padilla M: Mesodermal alterations induced by hypervitaminosis A. J Embryol Exp Morphol 15:261-269, 1966

32. Marin-Padilla M: Morphogenesis of anencephaly and related malformations. Curr Top Pathol 51:145-174, 1970

33. Marin-Padilla M: Morphogenesis of experimental encephalocele (Cranioschisis occulta). J Neurol Sci 46:83-99, 1980

34. Marin-Padilla M: Notochordal-basichondrocranium relationships: abnormalities in experimental axial skeletal (dysraphic) disorders. J Embryol Exp Morphol 53:15-38, 1979

35. Marin-Padilla M: The tethered cord syndrome: developmental considerations, in Holtzmann RNN, Stein BM (eds): The 
Tethered Spinal Cord. New York: Thieme-Stratton, 1985 pp 3-13

36. Marin-Padilla M, Marin-Padilla TM: Morphogenesis of experimentally induced Arnold-Chiari malformation. J Neurol Sci 50:29-55, 1981

37. McGuire EJ: The innervation and function of the lower urinary tract. J Neurosurg 65:278-285, 1986

38. McGuire EJ, Woodside JR, Borden TA, Weiss RM: Prognostic value of urodynamic testing in myelodysplastic patients. $\mathbf{J}$ Urol 126:205-209, 1981

39. McLone DG, Knepper PA: Role of complex carbohydrates and neurulation. Pediatr Neurosci 12:2-9, 1985-1986

40. McLone DG, Mutluer S, Naidich TP: Lipomeningoceles of the conus medullaris, in Karger S (ed): Concepts in Pediatric Neurosurgery. Basel: Karger, 1982, pp 171-177

41. McLone DG, Naidich TP: Laser resection of fifty spinal lipomas. Neurosurgery 18:611-615, 1986

42. McLone DG, Naidich TP: Spinal dysraphism: experimental and clinical, in Holtzman RN, Stein BM (eds): The Tethered Spinal Cord. New York: Thieme-Stratton, 1985, pp 14-28

43. McLone DG, Suwa J, Collins JA, Poznaski S, Knepper PA: Neurulation: biochemical and morphological studies on primary and secondary neural tube defects. Concepts Pediatr Neurosurg 4:15-29, 1983

44. Morriss-Kay GM, Crutch B: Culture of rat embryos with beta-D-xyloside: evidence of a role for proteoglycans in neurulation. J Anat 134:491-506, 1982

45. Müller F, O'Rahilly R: The development of the human brain, the closure of the caudal neuropore, and the beginning of secondary neurulation at stage 12. Anat Embryol (Berl) 176:413-430, 1987

46. O'Rahilly R, Meyer DB: The timing and sequence of events in the development of the human vertebral column during the embryonic period proper. Anat Embryol (Berl) 157:167-176, 1979

47. O'Shea KS, Kaufman MH: Phospholipase C-induced neural tube defects in the mouse embryo. Experientia 36:12171219,1980

48. Pang D: Commentary to the article: asymptomatic lumbosacral lipomas - a natural history study, by Wykes V, Desai D, and Thompson DNP. Childs Nerv Syst 28:1741-1742, 2012

49. Pang D: Electrophysiological monitoring for tethered cord surgery, in Yamada S (ed): Tethered Cord Syndrome, ed 2. New York: Thieme Medical Publishers, 2010, pp 199-209

50. Pang D: Intraoperative neurophysiology of the conus medullaris and cauda equina. Childs Nerv Syst 26:411-412, 2010

51. Pang D: Spinal cord lipoma, in Batjer H, Loftus C (eds): Textbook of Neurological Surgery. Philadelphia: Lippincott, Williams and Wilkins, 2002, pp 896-915

52. Pang D: Spinal cord lipomas, in Pang D (ed): Disorders of the Pediatric Spine. New York: Raven Press, 1995, pp 175-201

53. Pang D: Tethered cord syndrome, in Hoffman HJ (ed): Advances in Pediatric Neurosurgery. Philadelphia: Hanley and Belfus, 1986, pp 45-79

54. Pang D: Total resection of complex spinal cord lipomas: how, why, and when to operate? Neurol Med Chir (Tokyo) 55:695-721, 2015

55. Pang D, Casey K: Use of an anal sphincter pressure monitor during operations on the sacral spinal cord and nerve roots. Neurosurgery 13:562-568, 1983

56. Pang D, Wilberger JE Jr: Tethered cord syndrome in adults. J Neurosurg 57:32-47, 1982

57. Pang D, Zovickian J, Moes GS: Retained medullary cord in humans: late arrest of secondary neurulation. Neurosurgery 68:1500-1519, 2011

58. Pang D, Zovickian J, Oviedo A: Long-term outcome of total and near-total resection of spinal cord lipomas and radical reconstruction of the neural placode: part I-surgical technique. Neurosurgery 65:511-529, 2009

59. Pang D, Zovickian J, Oviedo A: Long-term outcome of total and near-total resection of spinal cord lipomas and radical reconstruction of the neural placode, part II: outcome analysis and preoperative profiling. Neurosurgery 66:253-273, 2010

60. Pang D, Zovickian J, Wong ST, Hou YJ, Moes GS: Surgical treatment of complex spinal cord lipomas. Childs Nerv Syst 29:1485-1513, 2013

61. Pierre-Kahn A, Lacombe J, Pichon J, Giudicelli Y, Renier D, Sainte-Rose C, et al: Intraspinal lipomas with spina bifida. Prognosis and treatment in 73 cases. J Neurosurg 65:756761, 1986

62. Pierre-Kahn A, Zerah M, Renier D, Cinalli G, Sainte-Rose C, Lellouch-Tubiana A, et al: Congenital lumbosacral lipomas. Childs Nerv Syst 13:298-335, 1997

63. Sathi S, Madsen JR, Bauer S, Scott RM: Effect of surgical repair on the neurologic function in infants with lipomeningocele. Pediatr Neurosurg 19:256-259, 1993

64. Schoenwolf GC: Histological and ultrastructural observations of tail bud formation in the chick embryo. Anat Rec 193:131-147, 1979

65. Schoenwolf GC: Histological and ultrastructural studies of secondary neurulation in mouse embryos. Am J Anat 169:361-376, 1984

66. Schut L, Bruce DA, Sutton LN: The management of the child with a lipomyelomeningocele. Clin Neurosurg 30:464-476, 1983

67. Stolke D, Zumkeller M, Seifert V: Intraspinal lipomas in infancy and childhood causing a tethered cord syndrome. Neurosurg Rev 11:59-65, 1988

68. Streeter GL: Factors involved in the formation of the filum terminale. Am J Anat 22:1-12, 1919

69. Sutton LN: Lipomyelomeningocele. Neurosurg Clin N Am 6:325-338, 1995

70. Talwalker VC, Datsur DK: Ectopic spinal cord myelomeningocele with tethering: a clinicopathological entity. Dev Med Child Neurol 16 (Suppl 32):159-160, 1974 (Abstract)

71. Toole BP: Glycosaminoglycans in morphogenesis, in Hay E (ed): Cell Biology of Extracellular Matrix. New York: Plenum Press, 1981, pp 229-294

72. Wykes V, Desai D, Thompson DNP: Asymptomatic lumbosacral lipomas - a natural history study. Childs Nerv Syst 28:1731-1739, 2012

73. Xenos C, Sgouros S, Walsh R, Hockley A: Spinal lipomas in children. Pediatr Neurosurg 32:295-307, 2000

\section{Disclosures}

The author reports no conflict of interest concerning the materials or methods used in this study or the findings specified in this paper.

\section{Supplemental Information \\ Online-Only Content}

Supplemental material is available with the online version of the article.

Supplemental Figures. https://thejns.org/doi/suppl/ 10.3171/2019.2.PEDS18390.

\section{Correspondence}

Dachling Pang: Great Ormond Street Hospital for Children, NHS Trust, London, United Kingdom. pangtv@aol.com. 